\title{
Body-induced vortical flows: a common mechanism for self-corrective trimming control in boxfishes
}

\author{
Ian K. Bartol ${ }^{1, *}$, Morteza Gharib ${ }^{2}$, Paul W. Webb ${ }^{3}$, Daniel Weihs ${ }^{4}$ and Malcolm S. Gordon ${ }^{5}$ \\ ${ }^{1}$ Department of Biological Sciences, Old Dominion University, Norfolk, VA 23529-0266, USA, \\ ${ }^{2}$ Options of Bioengineering and Aeronautics, California Institute of Technology, Pasadena, CA 91125, USA, \\ ${ }^{3}$ School of Natural Resources and Department of Biology, University of Michigan, Ann Arbor, MI 48109, USA, \\ ${ }^{4}$ Department of Aerospace Engineering, Technion, Haifa, 3200, Israel and ${ }^{5}$ Department of Ecology and Evolutionary \\ Biology, University of California, Los Angeles, CA 90095-1606, USA
}

*Author for correspondence (e-mail: ibartol@odu.edu)

Accepted 22 October 2004

\begin{abstract}
Summary
Boxfishes (Teleostei: Ostraciidae) are marine fishes having rigid carapaces that vary significantly among taxa in their shapes and structural ornamentation. We showed previously that the keels of the carapace of one species of tropical boxfish, the smooth trunkfish, produce leading edge vortices (LEVs) capable of generating self-correcting trimming forces during swimming. In this paper we show that other tropical boxfishes with different carapace shapes have similar capabilities. We conducted a quantitative study of flows around the carapaces of three morphologically distinct boxfishes (spotted boxfish, scrawled cowfish and buffalo trunkfish) using stereolithographic models and three separate but interrelated analytical approaches: digital particle image velocimetry (DPIV), pressure distribution measurements, and force balance measurements. The ventral keels of all three forms produced LEVs that grew in circulation along the bodies, resembling the LEVs produced around deltawinged aircraft. These spiral vortices formed above the

In the spotted boxfish, which is largely trapezoidal in cross section, consistent dorsal vortex growth posterior to the eye ridge was also present. When all three boxfishes were positioned at various yaw angles, regions of strongest concentrated vorticity formed in far-field locations of the carapace compared with near-field areas, and vortex circulation was greatest posterior to the center of mass. In general, regions of localized low pressure correlated well with regions of attached, concentrated vorticity, especially around the ventral keels. Although other features of the carapace also affect flow patterns and pressure distributions in different ways, the integrated effects of the flows were consistent for all forms: they produce trimming self-correcting forces, which we measured directly using the force balance. These data together with previous work on smooth trunkfish indicate that body-induced vortical flows are a common mechanism that is probably significant for trim control in all species of tropical boxfishes.
\end{abstract} keels and increased in circulation as pitch angle became more positive, and formed below the keels and increased in circulation as pitch angle became more negative. Vortices also formed along the eye ridges of all boxfishes.
Key words: vortex, boxfish, leading edge vortices (LEV), digital particle image velocimetry (DPIV), stability, pressure, pitch, yaw.

\section{Introduction}

The boxfishes (Teleostei: Ostraciidae) are marine, mostly shallow-water, mostly tropical reef-dwelling fishes that have the anterior $2 / 3$ to $3 / 4$ of their bodies encased in rigid bony carapaces consisting of hexagonal plates (or scutes; Tyler, 1980). These fishes swim by employing coordinated movements of their five fins. A detailed study of swimming dynamics and kinematics in one species, the spotted boxfish Ostracion meleagris, revealed that it uses distinct gaits to produce surprisingly constant costs of transport over a wide range of speeds (Gordon et al., 2000; Hove et al., 2001). Although they appear ungainly, boxfishes are capable of swimming rapidly $\left[>6\right.$ body lengths $\left.(B L) \mathrm{s}^{-1}\right]$, can spin around with a minimal turning radius, and hold precise control of their positions and orientations (Walker, 2000; Hove et al., 2001). The demonstration of some of the smallest amplitude recoil movements associated with swimming among fishes is one of the most notable qualities of boxfishes (Hove et al., 2001). In short, these fishes are remarkably stable, i.e. they can maintain desired swimming trajectories during steady and intentionally unsteady motions of maneuvers while being affected by both external and self-generated perturbations.

The bony carapaces of different species of boxfishes vary significantly in their cross-sections, longitudinal shapes and structural ornamentation, with some boxfishes having horns, 
spikes and pointed extensions of the carapace. The carapaces are generally not streamlined, but have longitudinal keels and shapes that appear to orient and control water flows over their surfaces in beneficial ways. Bartol et al. (2003) determined that the carapace of one boxfish, the smooth trunkfish Lactophrys triqueter, plays an integral role in the hydrodynamic stability of swimming. The ventro-lateral keels of this species of boxfish, which is broadly triangular in cross-section, produce leading edge vortical flows that increase in magnitude when the carapace pitches and yaws at greater angles. The vortices are strongest at posterior regions of the carapace and produce self-correcting trimming forces that probably help $L$. triqueter maintain smooth swimming trajectories in turbulent environments.

In this paper, we address a related question: do other boxfishes having different carapace morphologies have similar self-correcting mechanisms? To make this determination, flows around the bodies of three morphologically distinct boxfishes were investigated. The species were: (1) the spotted boxfish Ostracion meleagris, which has a trapezoidal crosssection and no significant ornamentation; (2) the scrawled cowfish Acanthostracion quadricornis, which has a pentagonal cross-section and has sharp anterior horns and bony projections extending from the ventral keels; and (3) the buffalo trunkfish Lactophrys trigonus, which has a triangular cross-section, a hump on its back, and ornamentation along the ventral keels (Fig. 1). Three separate but interrelated techniques were employed to investigate flow development along the carapaces (Bartol et al., 2003). For each approach, anatomically exact, stereolithographic models were used. The three techniques
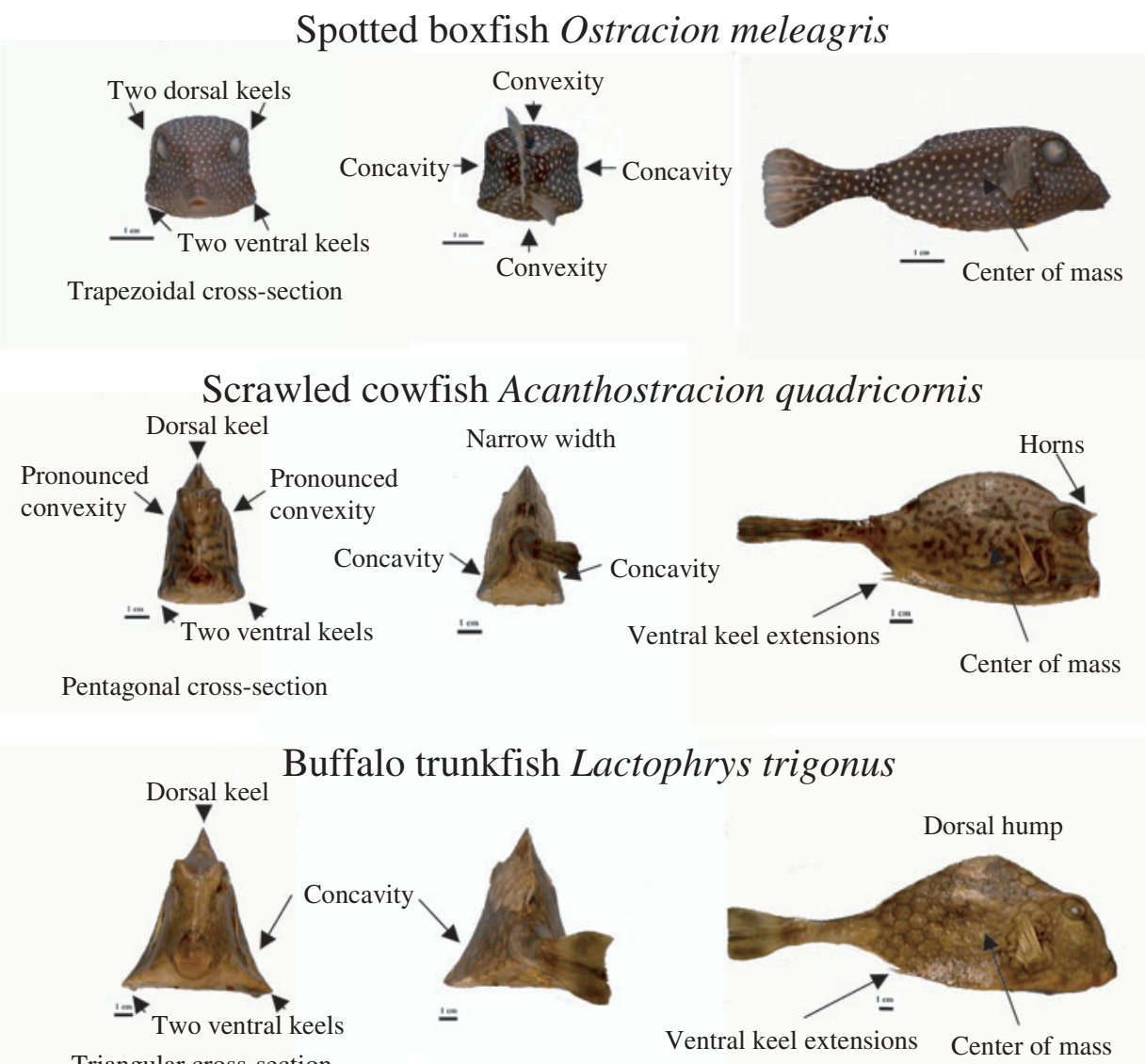

Triangular cross-section

Ventral keel extensions Center of mass
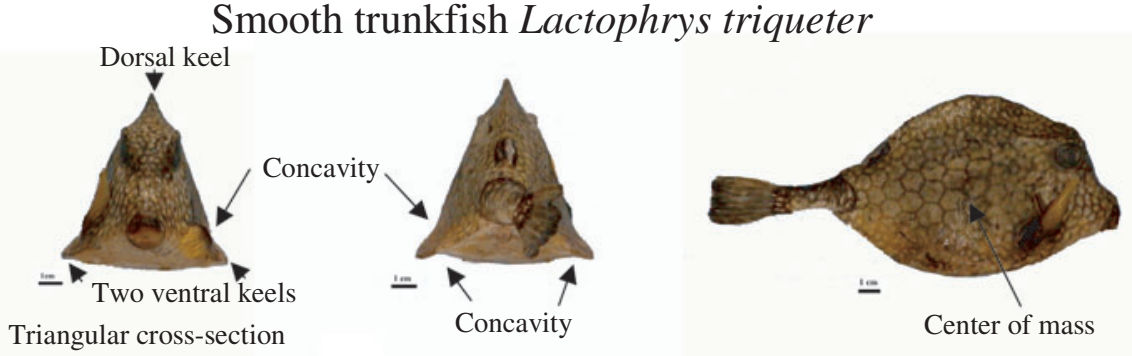

Fig. 1. Anterior, posterior, and lateral views of a spotted boxfish, scrawled cowfish, buffalo trunkfish, and smooth trunkfish. The smooth trunkfish was examined in Bartol et al. (2003). Some distinguishing features of each boxfish are highlighted in the figure. Scale bars, $1 \mathrm{~cm}$. 
were: (1) digital particle image velocimetry (DPIV), which provides global data on flow structures at selected planar sections along the carapaces; (2) pressure distribution measurements, which provide information on conditions at the surface of the carapaces, a region that is difficult to resolve using DPIV; and (3) force balance measurements, which provide an integrated measurement of the forces and moments acting on the carapaces.

\section{Materials and methods \\ Model construction}

An Hawaiian spotted boxfish Ostracion meleagris Shaw [12.1 cm total length $(T L), 48.5 \mathrm{~g}$ ] and two Puerto Rican boxfishes, a buffalo trunkfish Lactophrys trigonus L. (23.4 cm TL, 261.2 g) and a scrawled cowfish Acanthostracion quadricornis L. (16.4 cm TL, $18.8 \mathrm{~g})$, were captured, frozen immediately, and shipped frozen to the University of California, Los Angeles (UCLA), where they were stored in a freezer at $-70^{\circ} \mathrm{C}$. Computed tomography (CT) scans were performed on still frozen $\left(-70^{\circ} \mathrm{C}\right)$ specimens with the dorsal and anal fins removed, the pectoral fins positioned flush against the body, and the caudal fin aligned with the longitudinal axis of the body. The scans and subsequent stereolithographic model construction were performed using procedures described in Bartol et al. (2003). To improve resolution of DPIV and force balance measurements and accommodate more ports in pressure experiments, the $12.1 \mathrm{~cm}$ spotted boxfish was enlarged to $17.0 \mathrm{~cm}$ during model construction. All proportions were kept constant.

\section{Digital particle image velocimetry (DPIV)}

The basic 2-D DPIV technique for flow field measurements is described in Willert and Gharib (1991) and Raffel et al. (1998), and a more detailed description of our techniques is included in Bartol et al. (2003). The models were positioned at pitch angles $\left( \pm 30^{\circ}\right.$ at $2^{\circ}$ intervals $)$ and yaw angles $\left(0-30^{\circ}\right.$ at $10^{\circ}$ intervals) in a water tunnel with a $30 \mathrm{~cm} \times 30 \mathrm{~cm} \times 100 \mathrm{~cm}$ test section (Model 503, Engineering Laboratory Design, Inc., Lake City, MN, USA). The pitch angle refers to the angle between free-stream flow and a line connecting the center of mass and the center of the caudal peduncle (centerline of fish). The yaw angle refers to the angle between free-stream flow and the dorsal keel (for those fishes with one keel) or a line equidistant between the dorsal keels (for the spotted boxfish). The water tunnel was seeded with $14 \mu \mathrm{m}$ silver-coated, hollow glass spheres (Potter Industries Inc., Carlstadt, NJ, USA) and set at a speed of $44 \mathrm{~cm} \mathrm{~s}^{-1}\left(1.9-2.7 B L \mathrm{~B} \mathrm{~s}^{-1}\right)$ for all experimental trials. The water tunnel also was set at lower and higher speeds periodically to confirm that DPIV results at the intermediate speed above were qualitatively applicable over the range of swimming speeds of the fish.

A Pulnix TM-1320 digital video camera (Sunnyvale, CA, USA; $30 \mathrm{~Hz}$ frame rate, $480 \times 768$ pixel frame size) with a $35 \mathrm{~mm}$ or $85 \mathrm{~mm}$ Nikkor lens (Nikon, USA) was positioned downstream of the working section to record oncoming flows.
A dual pulsed ND:YAG laser (New Wave Research, Inc., Fremont, CA, USA; pulse duration $=0.02 \mathrm{~ms}$, pulse separation $=1 \mathrm{~ms}$ ), a series of front-surface mirrors, and a cylindrical lens were used to generate and align an illuminated sheet $\sim 1.0 \mathrm{~mm}$ thick. The laser sheet was projected underneath the water tunnel in a transverse $(Y Z)$ plane. The laser sheet was adjusted to illuminate planes in the wake of the models $(5 \mathrm{~cm}$ downstream from the caudal fin) and at five locations along the carapace: the eye ridge, the point of maximum girth, the midpoint between the point of maximum girth and the posterior edge of the carapace, the posterior edge of the carapace, and the caudal peduncle.

Using PixelFlow ${ }^{\mathrm{TM}}$ software (FG Group LLC, San Marino, CA, USA) and a customized timing box, we phase-locked the dual laser and a frame grabber to the video camera, and collected 30 paired images at each pitch/yaw angle for all planar locations described above. In all images, except those collected in the wake, the model was subtracted out of the field of view prior to processing. The displacement of particles within interrogation windows [ $32^{2}$ pixels with a 16 pixel offset] comprising the paired images was calculated by crosscorrelation (Willert and Gharib, 1991). Mean velocity and mean vorticity fields were determined for each trial. Circulation of regions of concentrated vorticity attached to the model were determined by carefully tracing along the outline of the model where the region of concentrated vorticity was present, tracing along an iso-vorticity contour of magnitude $2 \mathrm{~s}^{-1}$ found external to the model, and integrating the vorticity within the selected area. For detached vortices in the wake, circulation was computed by integrating vorticity within an iso-vorticity contour of magnitude $2 \mathrm{~s}^{-1}$ surrounding each vortex. Tracings and circulation calculations were performed using PixelFlow software.

\section{Pressure measurements}

Surface pressures along the boxfish carapaces were measured at multiple locations. Series of 36-48 holes, most often aligned in dorso-ventral transects at intervals along the carapaces, were drilled in sections of models that were fabricated in halves. It was necessary to drill some ports below the ventral keel slightly out of alignment with respect to other ports along a transect so as not to damage the delicate ventral keel extensions and/or provide viable channels for tubing. Urethane tubing $(0.068 \mathrm{~cm}$ i.d., $0.129 \mathrm{~cm}$ o.d.) was inserted into the holes and glued in place so that it was flush with the surface of the models. The tubing was threaded through a rod attached to the posterior section of the models, and the model halves were glued together. The rod was used to mount the models caudally to a sting in a $61 \mathrm{~cm}$ wind tunnel (model 407, Engineering Laboratory Design, Inc., Lake City, MN, USA). Tubing exiting the models was connected to a Scanivalve 48-channel rotating pressure scanner (Scanivalve Inc., Liberty Lake, WA, USA) and a barocel electric manometer and capacitative differential pressure sensor (Barocel Datametrics, Wilmington, MA, USA). Static pressure $\left(\mathrm{N} \mathrm{m}^{-2}\right)$ at each of the ports was expressed relative to static pressure at a tunnel wall port. 
Data were collected while each model was positioned at $2^{\circ}$ increments for pitch angles of -30 to $+30^{\circ}$. For the angles considered, data were acquired at $100 \mathrm{~Hz}$ for $10 \mathrm{~s}$ using LabVIEW software (National Instruments, Inc., Austin, TX, USA). Wind tunnel speed was set according to the Reynolds number considered in water tunnel trials. Mean pressure coefficients $\left(C_{\mathrm{P}}\right)$ were calculated at each port for each pitch angle using the equation described in Bartol et al. (2003).

\section{Force measurements}

Each of the three models was mounted to a sting in a large water tunnel (test section $61 \mathrm{~cm} \times 46 \mathrm{~cm} \times 244 \mathrm{~cm}$ ), and force measurements were collected using an in-house force balance (Lisoski, 1993) containing two load cells for measuring lift and one for measuring drag (Interface, Inc. Scottsdale, AZ, USA). Force data were collected in $2^{\circ}$ increments from pitch angles of at least -30 to $+30^{\circ}$. Flow speed during trials was identical to that considered in DPIV experiments $\left(44 \mathrm{~cm} \mathrm{~s}^{-1}\right.$; i.e. 1.9-2.7 $B L \mathrm{~s}^{-1}$ ). At each pitch angle considered, data output was recorded at $200 \mathrm{~Hz}$ for $10 \mathrm{~s}$ using a Dash 8 Series data recorder (Astro-Med, Inc., West Warwick, RI, USA). Coefficients of drag $\left(C_{\mathrm{D}}\right)$, lift $\left(C_{\mathrm{L}}\right)$ and pitch moment $\left(C_{\mathrm{M}}\right)$ were calculated using equations described in Bartol et al. (2003).

\section{Results \\ Digital particle image velocimetry \\ Ventral keels - pitch}

When all three boxfish models were positioned at positive pitch angles, regions of concentrated vorticity (leading edge vortices; LEVs) began to develop near the anterior edges of the ventral keels at a longitudinal location corresponding to the eye ridge (Fig. 2). These regions of concentrated vorticity intensified in peak vorticity and circulation posteriorly along the ventral keels until two well-developed, counter-rotating vortices ultimately formed above the ventral keels at the posterior edge of the carapace. These ventral vortices left the body at the caudal peduncle, where vortices increased in terms of circulation and peak vorticity relative to attached vortices at the posterior edge of the carapace (Fig. 2). In the wake, ventral vortices either merged with dorsal vortices or were in the process of merging. Clockwise rotation was present on the left side of the carapaces (when viewed from the rear), while counterclockwise rotation was present on the right side of the carapaces, resulting in the development of a downwash of flow in the wake.

At negative pitch angles, regions of concentrated, attached vorticity also began to develop around the ventral keels at a longitudinal location corresponding to the eye ridge in all three boxfishes (Fig. 3). As was the case for positive pitch angles, circulation and peak vorticity of ventral, concentrated, attached vorticity increased posteriorly, developing into two counterrotating vortices at the posterior edge of the carapace and ultimately leaving the body close to the caudal peduncle. However, while regions of concentrated vorticity developed above the ventral keels at positive pitch angles, regions of concentrated vorticity formed below the ventral keels at negative pitch angles (Fig. 3). Furthermore, when compared to flow patterns at positive pitch angles, vortical flow rotation reversed in direction (i.e. vortices around the right ventral keel were rotated counterclockwise when viewed from the rear at positive pitch angles but clockwise at negative pitch angles). Therefore, an upwash of flow developed in the wake at negative pitch angles.

Although the location and pattern of vortex development did not change appreciably for different positive pitch angles or for different negative pitch angles, the magnitude of vortex circulation and peak vorticity did. At a pitch angle close to $0^{\circ}$, peak vorticity and circulation of ventral vortices were lowest for all three boxfishes (Fig. 4). At $0^{\circ}$ the direction of vortex rotation was similar to that at positive pitch angles, and a downwash of flow was observed in velocity vector plots. As the carapace was positioned at increasingly more positive pitch angles, ventral vortices increased in peak vorticity and circulation. Similarly, as the carapace was positioned at increasingly more negative pitch angles, ventral vortices increased in peak vorticity and circulation.

In the scrawled cowfish and buffalo trunkfish, the cores of vortices always formed approximately above or below the ventral keel extensions at the posterior edge of the carapace at positive and negative pitch angles, respectively (Fig. 4). However, in the spotted boxfish, which lacks ventral keel extensions, the cores of regions of concentrated vorticity were not always directly above or below the ventral keel at the posterior edge of the carapace.

\section{Dorsal keels - pitch}

Regions of attached, concentrated vorticity formed dorsally around the eye ridges of all three boxfishes at positive pitch angles (Fig. 2). At the eye ridge dorso-ventral transect, dorsal regions of concentrated vorticity were greater in circulation and peak vorticity than ventral regions of concentrated vorticity. However, at the posterior edge of the carapace where both dorsal and ventral attached vortices were strongest in circulation, dorsal vortices had less circulation and lower peak vorticity than ventral vortices. Some regions of weaker, concentrated, attached vorticity also formed around the eye ridges at negative pitch angles (Fig. 3).

In the spotted boxfish, dorsal regions of attached vorticity grew consistently in terms of peak vorticity and circulation posterior to the eye ridges both at positive and negative pitch angles (Figs 2, 3). Although there was some migratory movement of concentrated vorticity around the dorsal keels at low positive pitch angles, dorsal vortices generally grew along and above the dorsal keels at positive pitch angles until two well-developed vortices ultimately formed at the posterior edge of the carapace. Dorsal vortex circulation growth was particularly pronounced in the posterior quarter of the carapace, sometimes even outpacing ventral vortex growth. At negative pitch angles, dorsal vortices also grew along the dorsal keels, but two well-developed vortices formed below the 

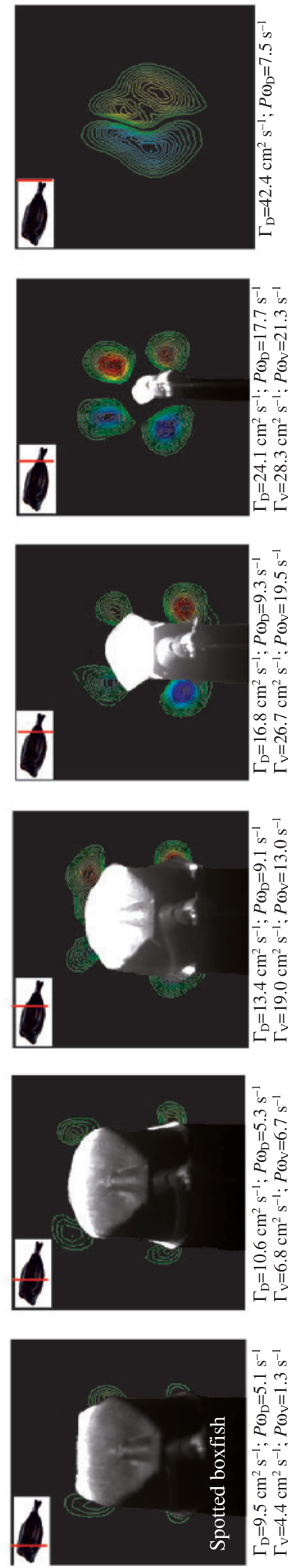
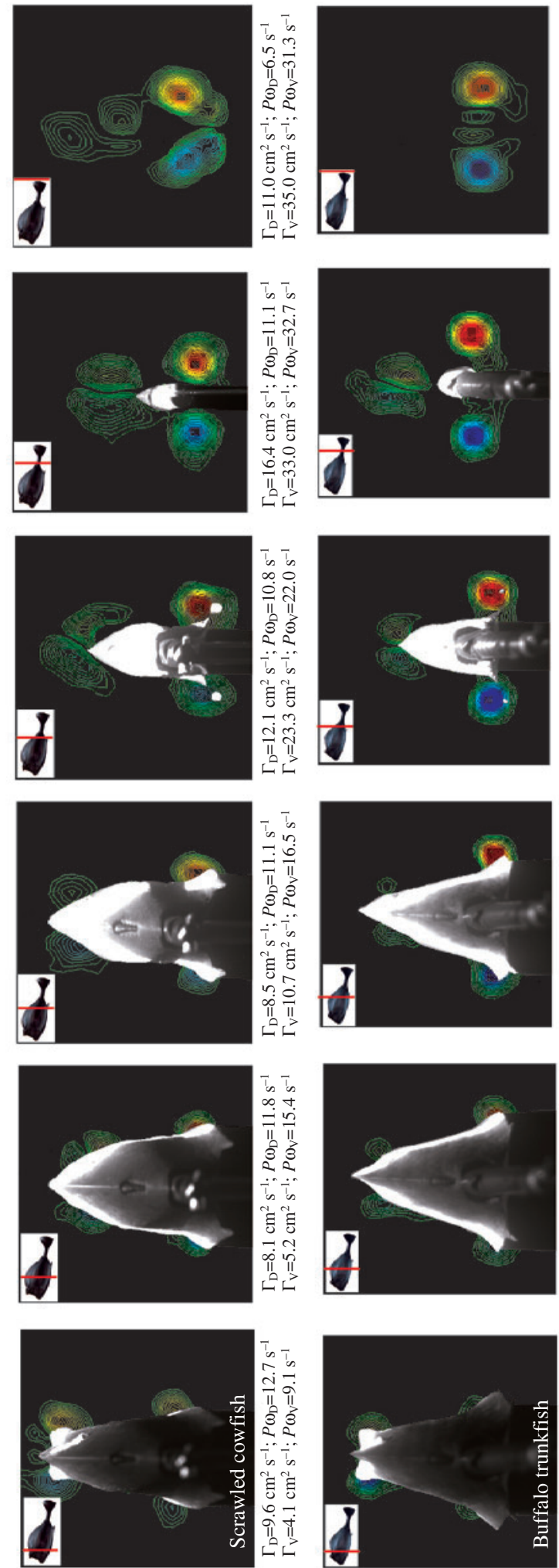

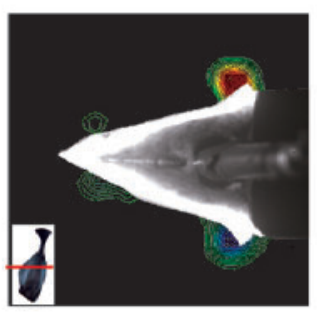

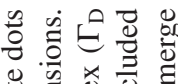

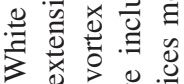

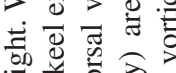

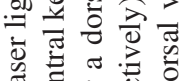

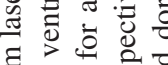

政

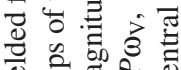

을

To

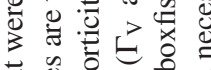

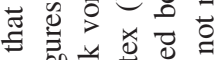

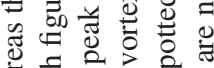

का

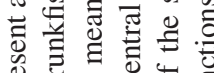

†

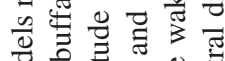

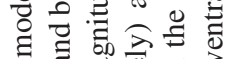

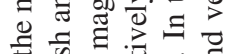

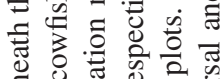

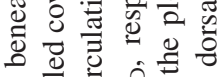

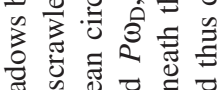

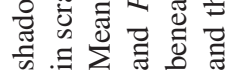

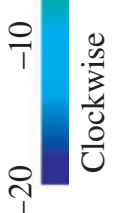

in

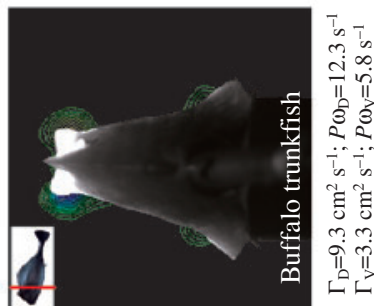

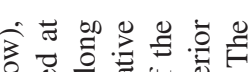

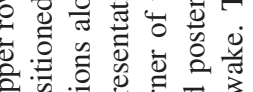

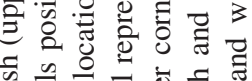

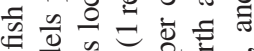

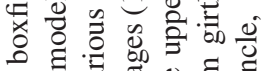

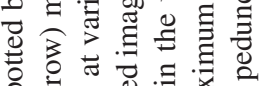
के

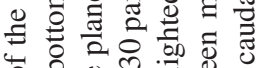

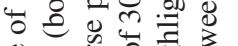

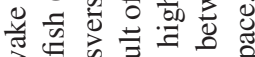

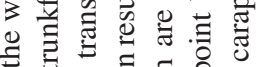

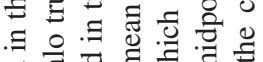

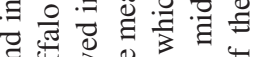

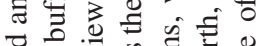

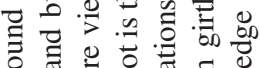
政

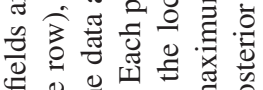

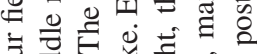

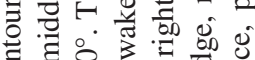
이의

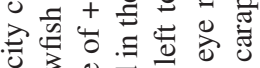
记

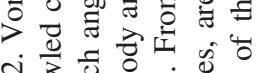

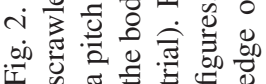



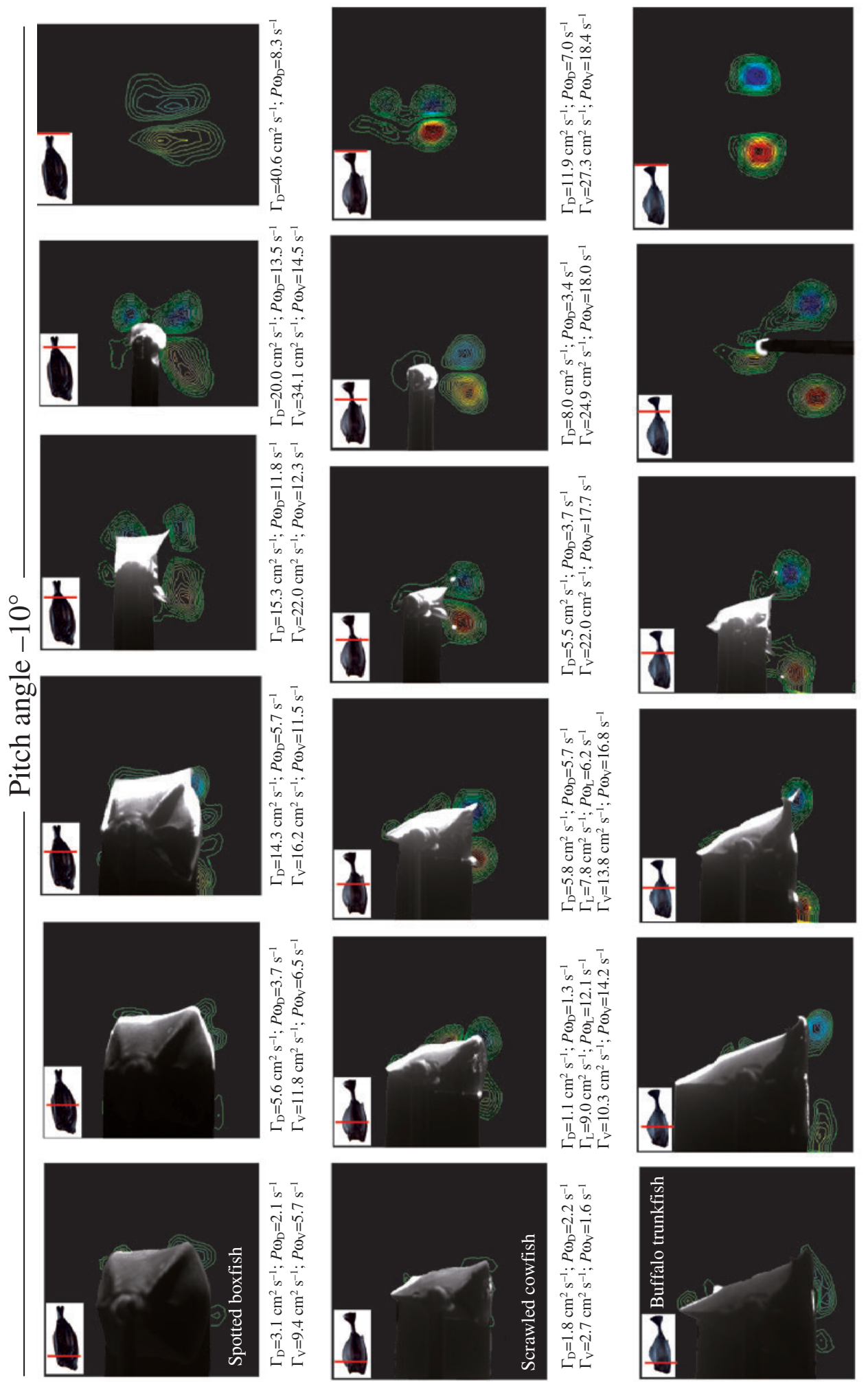
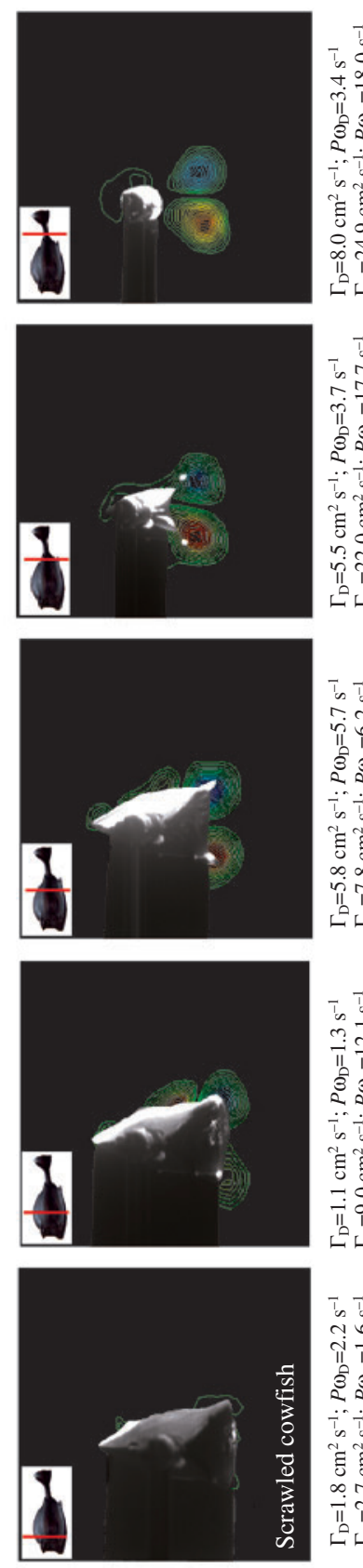
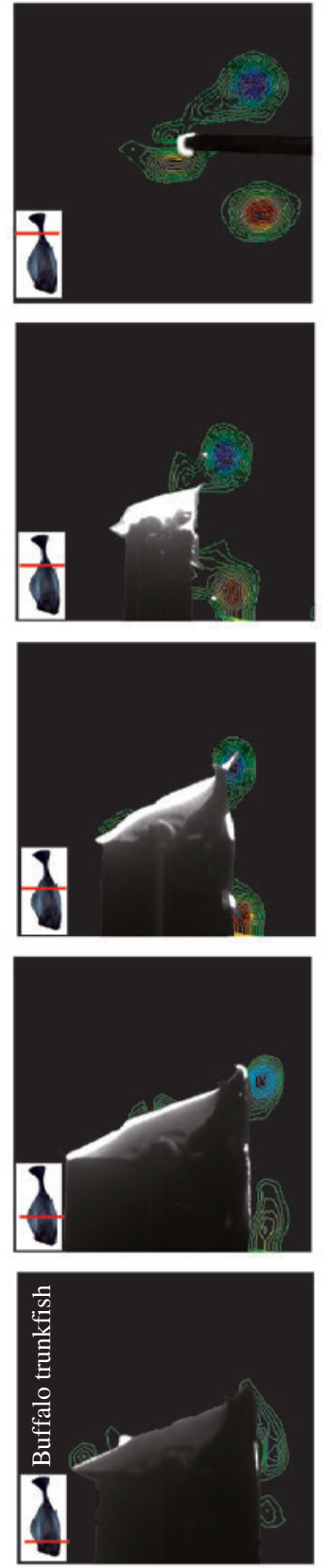

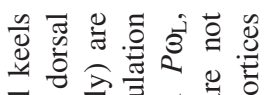

정

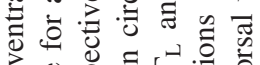

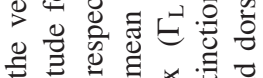

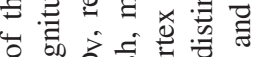

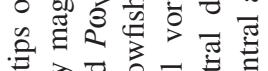

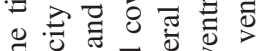

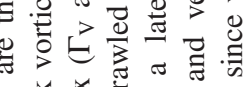

象善

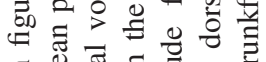

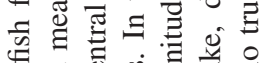

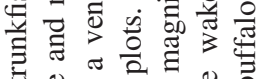

을

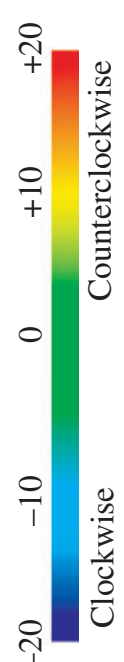

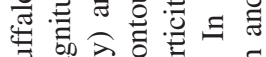

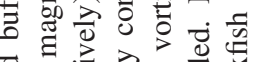

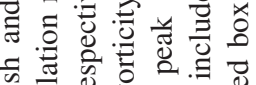

कृ

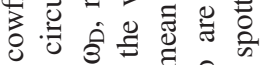

马

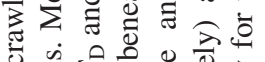

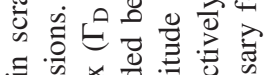

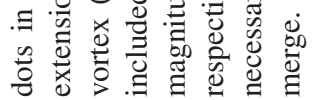

ใิํำ

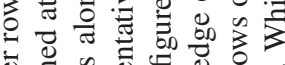

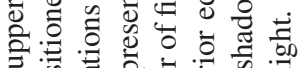

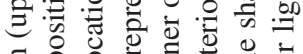

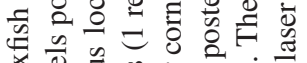

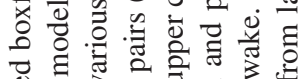

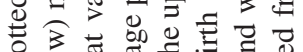

की

‡

प艹

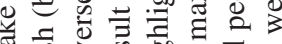

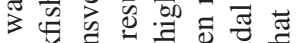

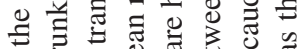

$. \Xi \Xi . \Xi$ 过

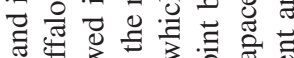

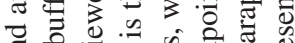

定 $\overrightarrow{0}$ 흥

흐

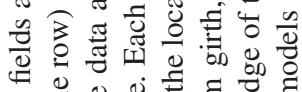

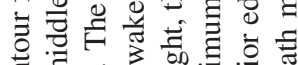

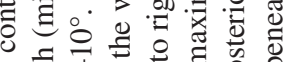

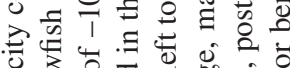

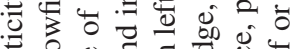

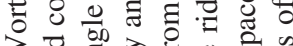

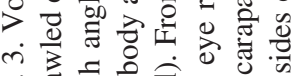

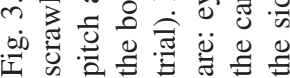



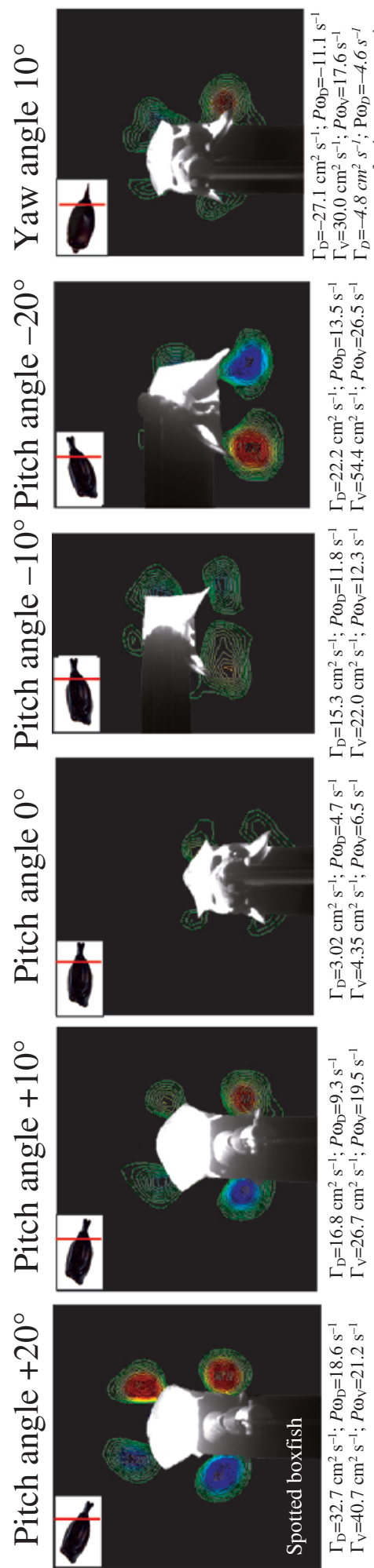
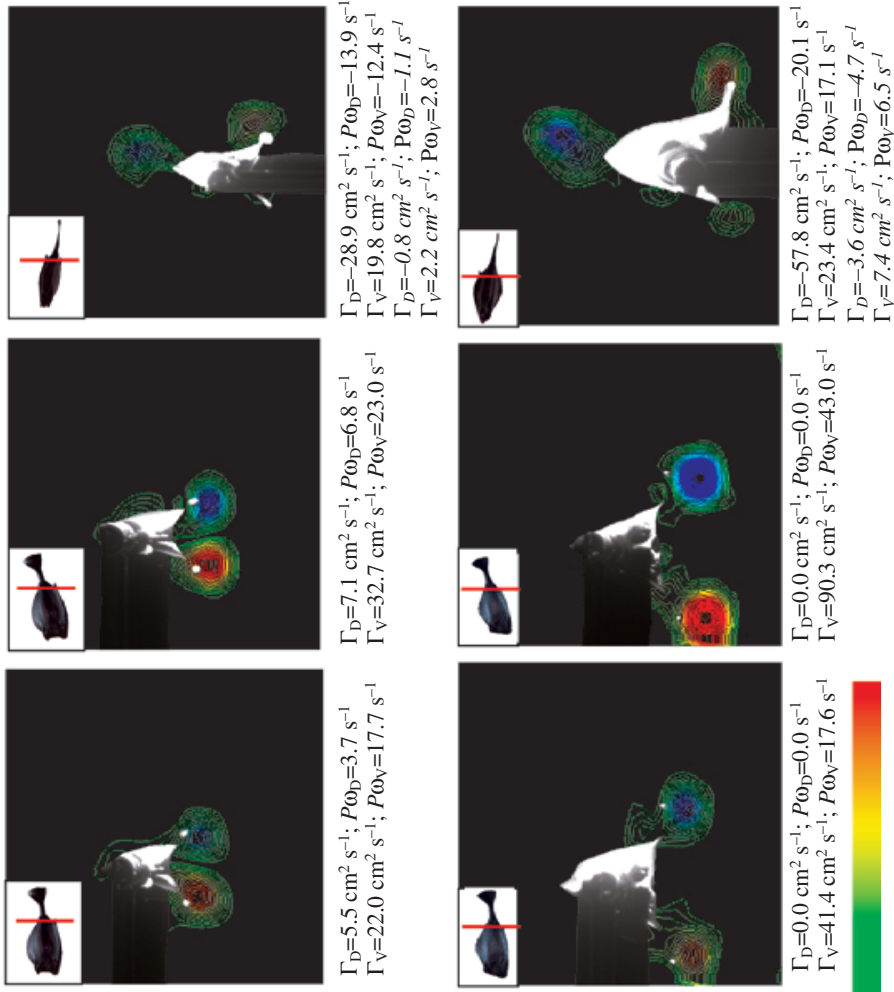

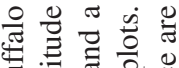
击 品 렬 흠 矛 - ธี के चे

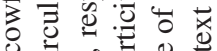
उ

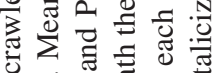

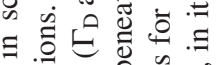

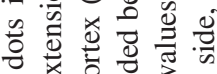

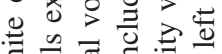

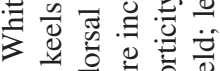
$\left.+\frac{\pi}{4}\right)^{\circ}$

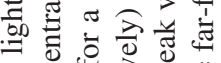
ப)

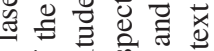
हี

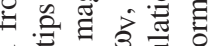

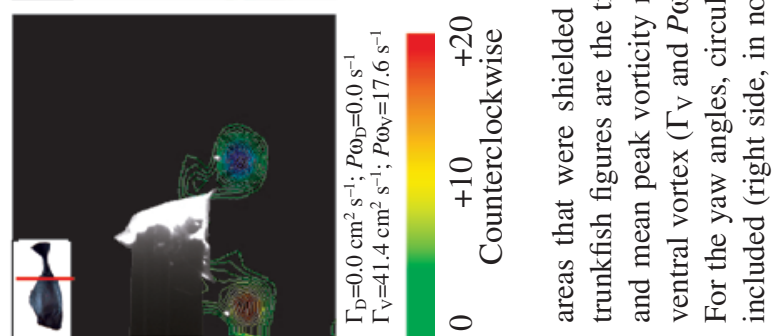
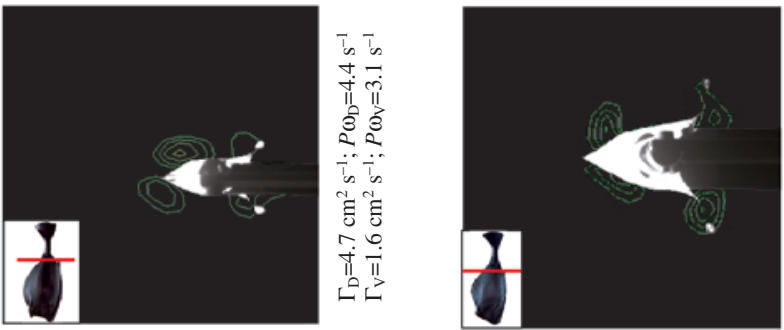

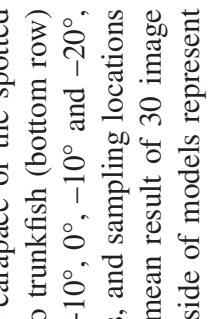
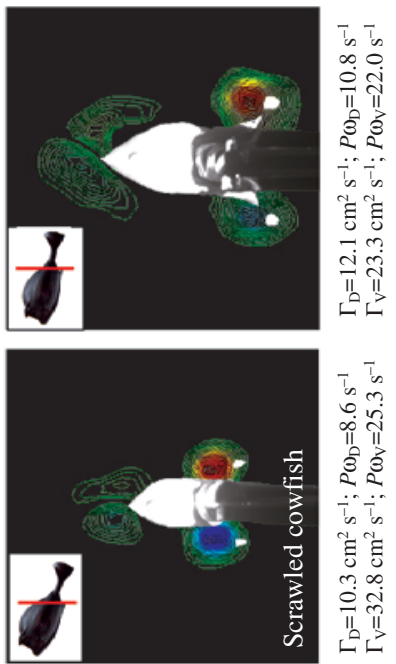

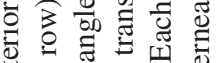

훙.

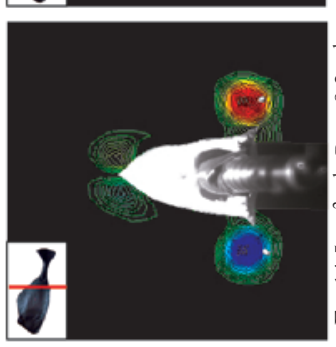

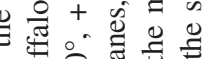

0 零

品 考

व.

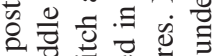

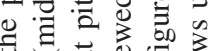

च ज्ञ

它施的

ส 8 용

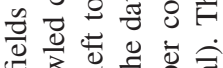

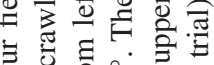

ठิ)

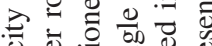

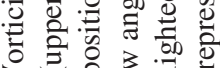

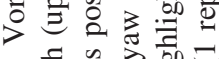

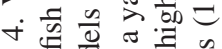

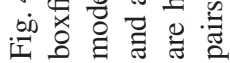


A

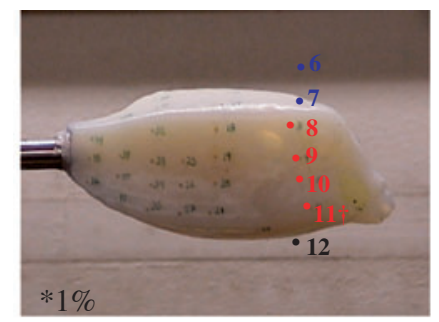

B
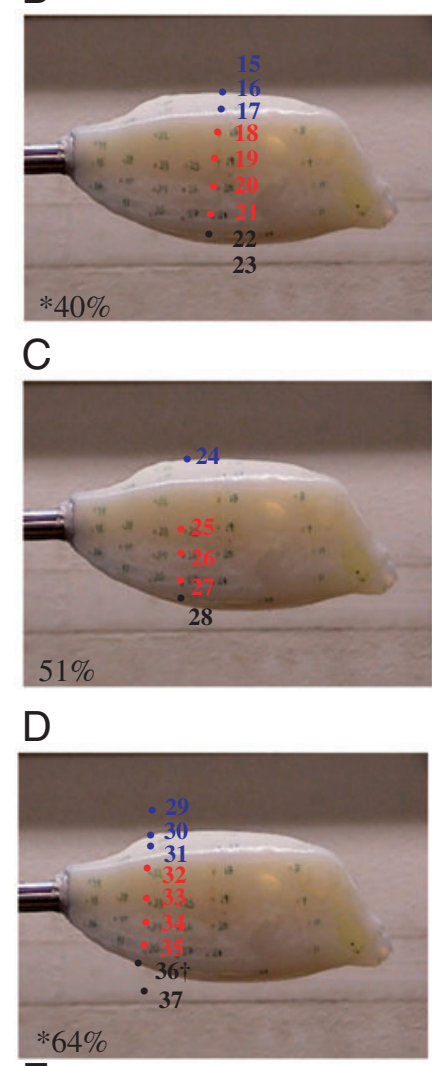

E

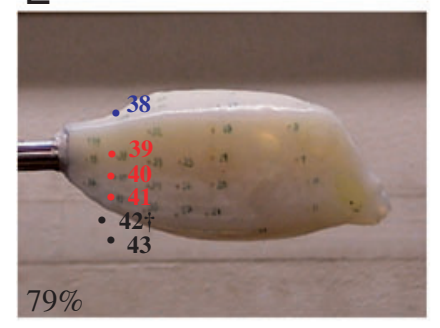

F

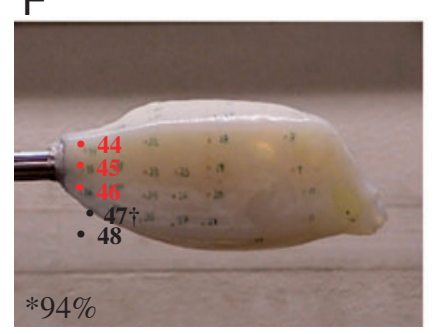

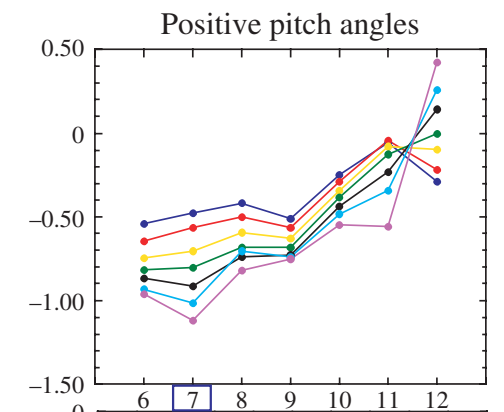
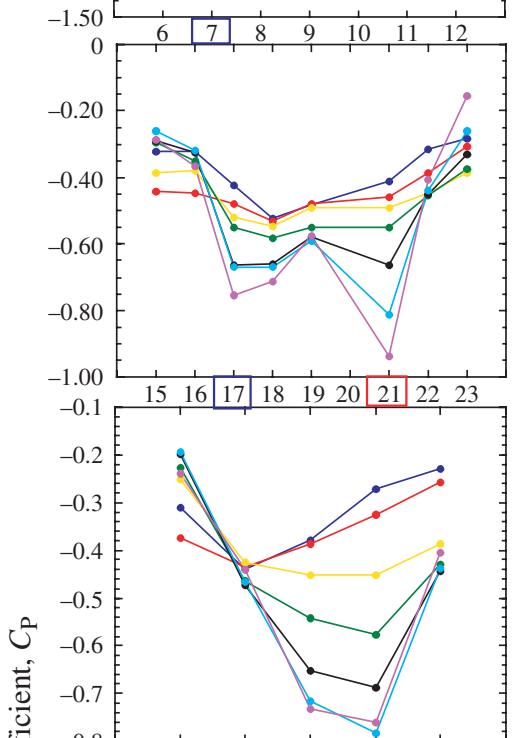

退
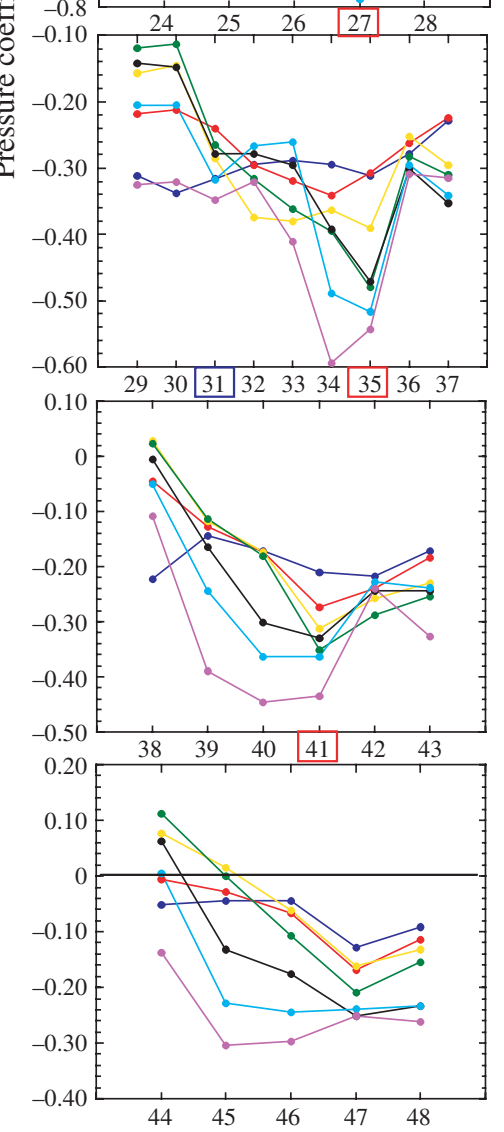

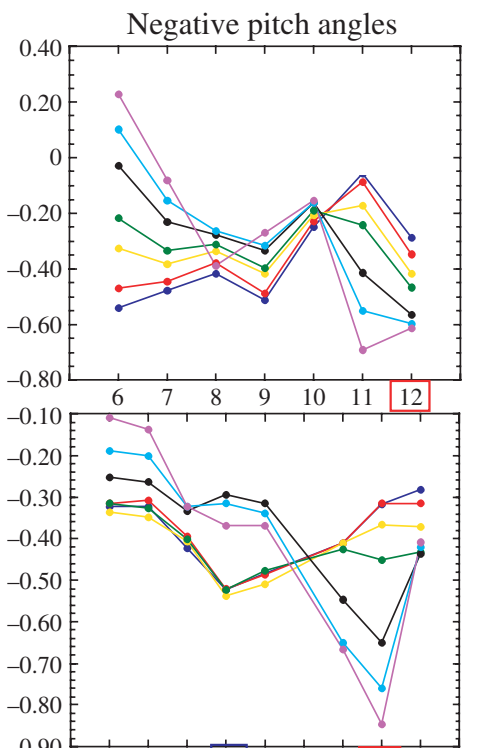

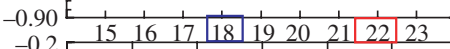
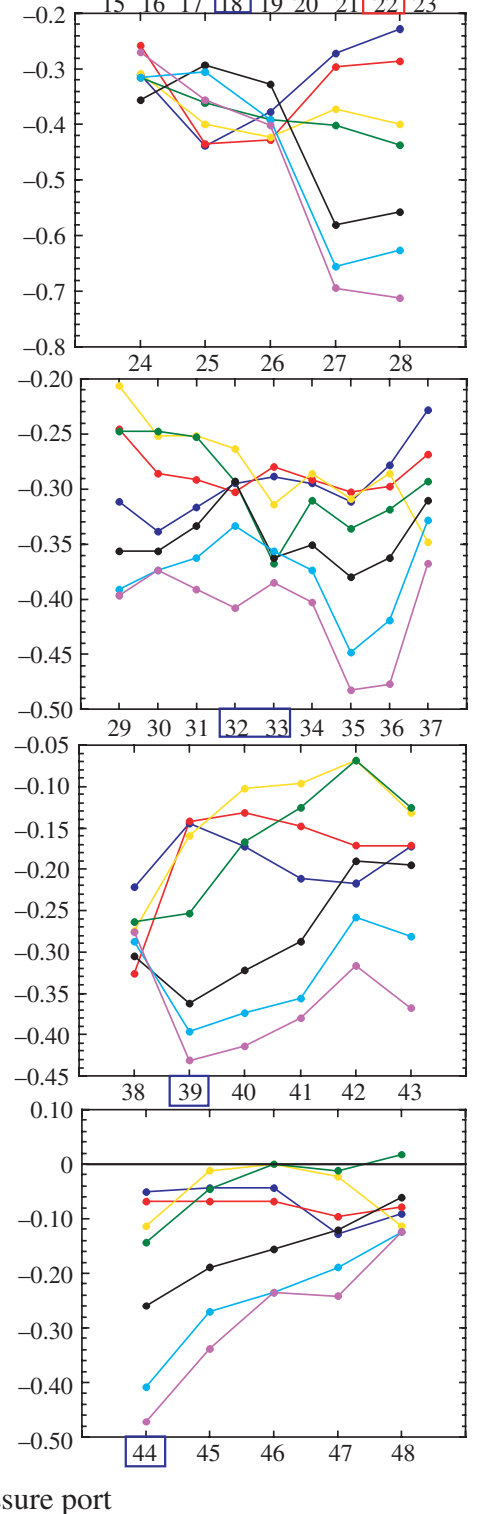

Pitch angle (deg.)

$-0$

+4 or -4

+10 or -10

+14 or -14

+20 or -20

+24 or -24

+30 or -30 
dorsal keels, reaching maximum strength at the posterior edge of the carapace. Peak vorticity and circulation of dorsal vortices increased with more positive and negative pitch angles (Fig. 4). At both positive and negative pitch angles, dorsal vortices always merged with ventral vortices in the wake of the spotted boxfish.

In the other boxfishes, dorsal vortex formation posterior to the eye ridge was less well defined. In the scrawled cowfish, regions of attached vorticity shed from the anterior horns/eye ridges persisted on either side of the dorsal keel at positive pitch angles (Fig. 2). These vortices remained relatively constant in circulation along the body, forming above lateral regions of high convexity, before increasing slightly along the posterior quarter of the carapace. At negative pitch angles, multiple regions of concentrated vorticity often developed in lateral locations (Fig. 3). The strongest region of concentrated vorticity frequently formed below the region of maximum convexity and merged with weaker dorsal regions of concentrated vorticity at the posterior edge of the carapace. No consistent increase in circulation of dorsal vortices with more positive or negative pitch angle was observed in the scrawled cowfish, but circulation of dorsal vortices was generally higher for positive compared with negative pitch angles (Fig. 4). At both positive and negative pitch angles, dorsal/lateral and ventral vortices were often in the process of merging at the wake sampling location (i.e. $5 \mathrm{~cm}$ from the caudal fin), but vortex unification was not always complete (Figs 2, 3).

In the buffalo trunkfish, regions of concentrated dorsal vorticity sometimes detached from the body and did not remain constant or grow in a consistent pattern posteriorly at either positive or negative pitch angles (Figs 2, 3). In fact, circulation and peak vorticity decreased frequently at certain locations along the carapace when moving antero-posteriorly. Not surprisingly, no consistent increase in circulation of dorsal vortices with more positive or negative pitch angle was observed in the buffalo trunkfish (Fig. 4). In most cases, ventral and dorsal vortices, if present, merged in the wake.

\section{Ventral and dorsal keels - yaw}

When the carapaces of each of the three boxfishes were positioned at yaw angles, regions of concentrated vorticity had

Fig. 5. Pressure coefficients $\left(\mathrm{C}_{\mathrm{p}}\right)$ plotted as a function of location along various dorso-ventral transects $(\mathrm{A}-\mathrm{F})$ on the spotted boxfish model positioned at positive (left) and negative (right) pitch angles. The locations of the pressure ports included in each graph are highlighted in images to the left of the graphs. The location of each dorso-ventral transect is expressed as a percentage of ventral keel length (measured from the anterior leading edge) in the lower lefthand corner of each image. Ports in blue, red and black are located on the dorsum, sides, and ventrum of the model, respectively. ${ }^{\dagger}$ Denotes ports that were slightly out of alignment with other ports along the dorso-ventral transect; * represent transects that were considered in DPIV studies. Blue and red rectangles highlight ports where local pressure minima were consistently detected at dorsal and ventral locations, respectively. An ambient pressure line $\left(C_{\mathrm{p}}=0\right)$ was included in the most posteriorly located transect $(\mathrm{F})$. greater circulation and peak vorticity in far-field regions of the carapace (i.e. portions of the carapace that are shielded somewhat from oncoming flow) than in near-field locations (i.e. portions of the carapace that are directly exposed to oncoming flow; Fig. 4). In general, one vortex formed along the dorsal keel and one vortex formed along the ventral keel of each boxfish at the far-field side of the carapace. Vortex development in these far-field locations began at anterior regions of the dorsal and ventral keels and grew along the body before detaching from the body at either the posterior edge of the carapace or caudal peduncle. In contrast to pitch angles where vortex rotation was in the same direction on a particular side of the carapace, dorsal and ventral vortices on the far-field side of the carapaces rotated in different directions. For example, when viewed from the rear of the carapace and when the far-field region is on the right side of the carapace, a clockwise and counterclockwise vortex developed around the dorsal and ventral keels, respectively (Fig. 4). As yaw angles increased from $0^{\circ}$, peak vorticity and circulation of far-field vortices intensified. As was the case with pitch angles, peak vorticity and circulation of attached vortices were always greatest at the posterior edge of the carapace, irrespective of angle.

\section{Pressure measurements}

\section{Terminology and figure format}

Throughout this section, dorso-ventral transects in Figs 5-7 will be referenced. These transects are expressed as percentages of distance along the ventral keel beginning at the anterior end. Transects that coincide with those considered in DPIV experiments are denoted by asterisks in the figures to facilitate cross-referencing with DPIV data. The term 'local pressure minimum' will be used throughout this section. A local pressure minimum refers to low pressure at a port relative to its immediate dorsal and ventral neighbors (if present) along a selected dorso-ventral transect.

\section{Ventral keels}

For all three boxfishes, local pressure minima were often detected near the ventral keels in locations where regions of attached, concentrated vorticity were observed, with the more acutely keeled scrawled cowfish and buffalo trunkfish having more detectable minima than the spotted boxfish. At positive pitch angles, local pressure minima generally were detected at ports just above the ventral keels. Ports where local pressure minima were consistently detected at positive pitch angles are highlighted in red boxes (see positive pitch angle column in Figs 5-7). The lack of local pressure minima at ports 11 and 46 in the spotted boxfish is likely the product of port misalignment (e.g. port 11 is more anterior than neighboring ports and port 46 is more posterior than its ventral neighbor, port 47). In the spotted boxfish and buffalo trunkfish, local low pressure was detected in lateral regions above the ventral keels, as well as regions just above the ventral keels, at positive pitch angles. For example, in the spotted boxfish, local low pressure was detected at pressure port 34 for pitch angles $\geqslant 24^{\circ}$ and 
A

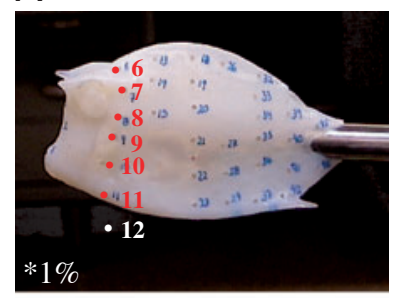

B

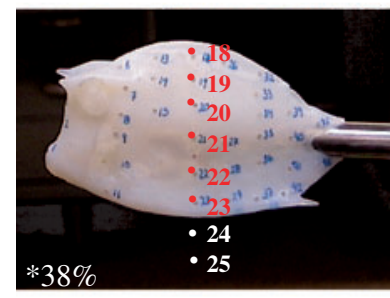

C

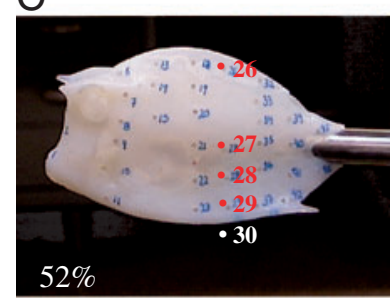

D

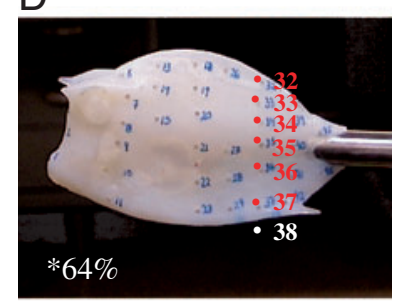

E

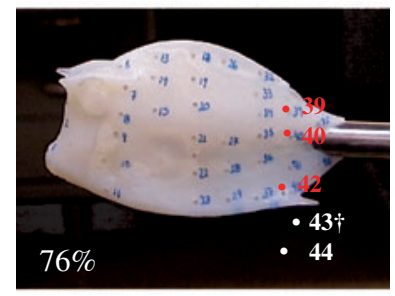

$\mathrm{F}$

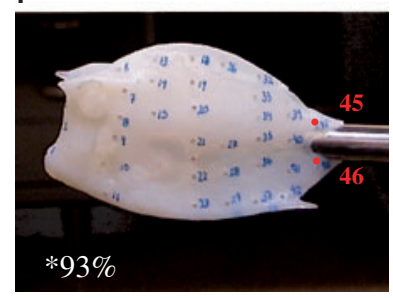

Positive pitch angles
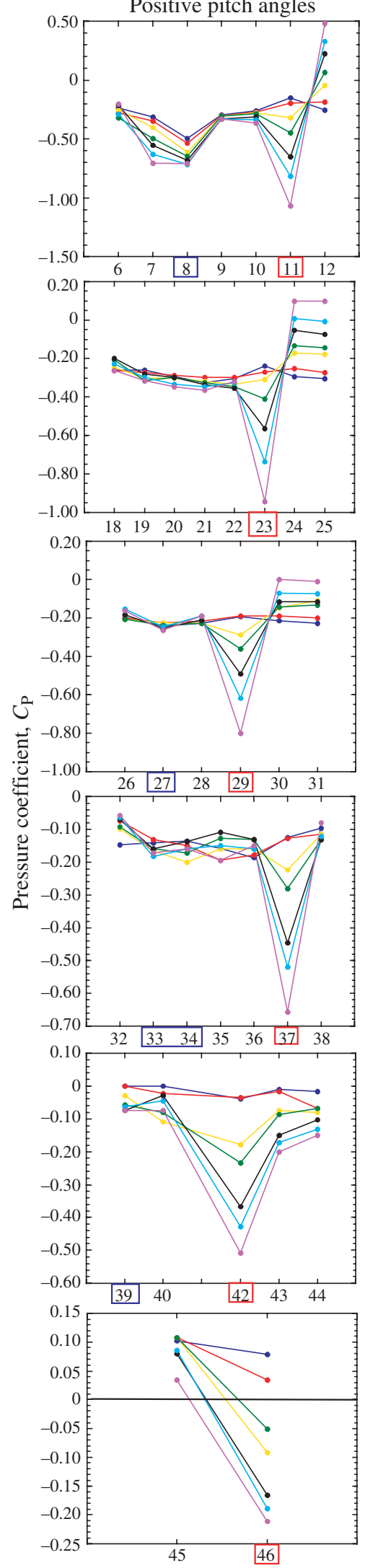

Negative pitch angles
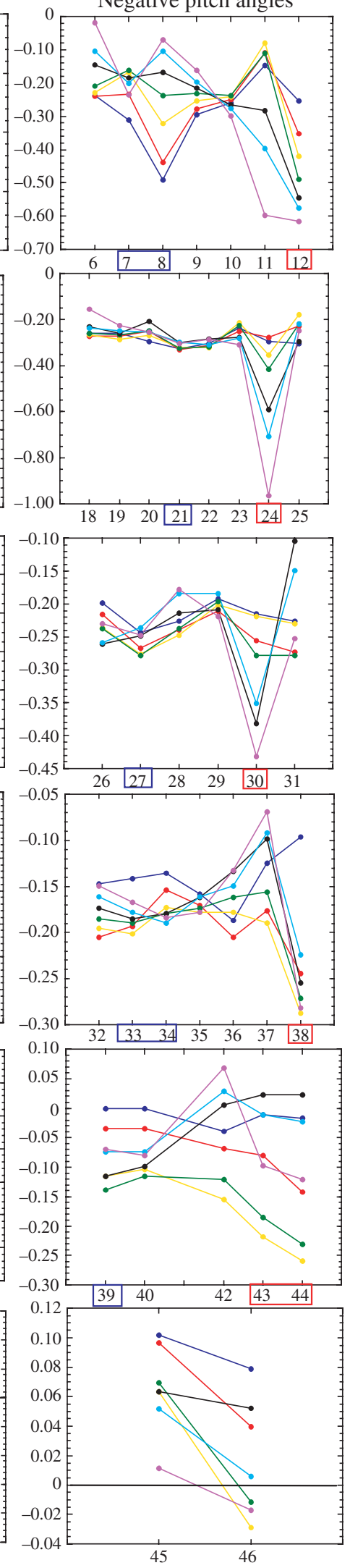

Pitch angle (deg.)

$-0$

+4 or -4

+10 or -10

+14 or -14

+20 or -20

+24 or -24

+30 or -30

Pressure port 
ports 40 and 45 for pitch angles $\geqslant 18^{\circ}$ (Fig. 5). In the buffalo trunkfish, local low pressure was observed at ports 35 and 41 at pitch angles $\geqslant 26^{\circ}$ (Fig. 7).

At negative pitch angles, local pressure minima generally were detected at ports just below the ventral keels, which is where regions of concentrated vorticity were detected. Ports where local pressure minima were consistently detected at negative pitch angles are highlighted in red boxes (see negative pitch angle column in Figs 5-7). The absence of local pressure minima below the ventral keel at various transects was often a product of misalignment (e.g. ports 36 and 42 in spotted boxfish and port 43 in buffalo trunkfish) or the absence of ports below the ventral keel (e.g. 93\% transect in scrawled cowfish, $87 \%$ transect in buffalo trunkfish). In the scrawled cowfish, local low pressures often were detected both at port 43 , which is located just below the ventral keel extension (see $76 \%$ transect), and port 44 , which is located near the midline of the ventrum in a region of high concavity. Low pressures at ports 43 and 44 were particularly pronounced at pitch angles of -10 and $-14^{\circ}$, where pressures were lower than at the other angles.

For all three boxfishes, pressure generally decreased at the ports above when pitch angles became more positive or more negative, just as regions of concentrated vorticity increased in circulation with increased pitch angle (Figs 5-7). Although peak vorticity and vortex circulation increased consistently along the ventral keels of the boxfishes from the anterior edge of the keel to the posterior edge of the carapace, pressure values at these locations did not decrease antero-posteriorly at either positive or negative pitch angles, and pressure was not lowest at posterior regions of the carapace. Instead pressure above/below ventral keels was most often lowest near maximum girth (see $40 \%$ transect for spotted boxfish, 38\% transect for scrawled cowfish, and $32 \%$ transect for buffalo trunkfish in Figs 5-7).

In general, pressure adjacent to the ventral keels at the posteriormost regions of the carapaces of the three boxfishes (where regions of concentrated vorticity were observed) was not above ambient levels for positive and negative pitch angles (i.e. pressure coefficients were $<0$ ). Examples are found in Figs 5-7 [see port 46 (positive pitch) and port 47 (negative pitch) for spotted boxfish; port 46 (positive pitch)

Fig. 6. Pressure coefficients $\left(C_{\mathrm{p}}\right)$ plotted as a function of location along various dorso-ventral transects $(\mathrm{A}-\mathrm{F})$ on the scrawled cowfish model positioned at positive (left) and negative (right) pitch angles. The locations of the pressure ports included in each graph are highlighted in images to the left of the graphs. The location of each dorso-ventral transect is expressed as a percentage of ventral keel length (measured from the anterior leading edge) in the lower lefthand corner of each image. Ports in red and white are located on the sides and ventrum of the model, respectively. ${ }^{\dagger}$ Denotes ports that were slightly out of alignment with other ports along the dorso-ventral transect; * represent transects that were considered in DPIV studies. Blue and red rectangles highlight ports where local pressure minima were consistently detected at dorsal and ventral locations, respectively. An ambient pressure line $\left(C_{\mathrm{p}}=0\right)$ was included in the most posteriorly located transect $(\mathrm{F})$. for scrawled cowfish; and port 47 (positive pitch) for buffalo trunkfish].

\section{Dorsal keels}

Correlations between localized low pressure and concentrated vorticity were less apparent in dorsal locations, where vortices were lower in circulation magnitude than in ventral locations. The most consistent correlations in dorsal locations occurred in the trapezoidal spotted boxfish, especially at higher pitch angles. At the $1 \%, 40 \%$ and $64 \%$ transects, local pressure minima were detected above the dorsal keel at positive pitch angles in regions where concentrated vorticity also was observed (see ports highlighted in blue in Fig. 5). Local pressure minima were not detected above the dorsal keel at the $51 \%$ and $94 \%$ transects because no ports were sampled just above the dorsal keel. At negative pitch angles, local pressure minima were detected along most transects where ports just below the dorsal keel were present (see ports highlighted in blue in Fig. 5). Interestingly, along the $40 \%$ transect local pressure minima were sometimes located at port 18 (below the dorsal keel) at certain positive pitch angles, and at port 17 (above the dorsal keel) at certain negative pitch angles. This finding is consistent with the observed migratory movement of concentrated vorticity in DPIV experiments.

In the scrawled cowfish, regions of concentrated vorticity formed around the eye ridges and at lateral locations along the carapace at positive/negative pitch angles. Local pressure minima were present near the eye ridges (see ports 7 and 8, Fig. 6). In lateral locations, there was evidence of local pressure minima at the $38-76 \%$ transects, but the minima were subtle relative to dorsal pressure minima detected in the spotted boxfish (see blue boxes, Fig. 6). At positive and negative pitch angles, regions of concentrated vorticity formed most consistently around the eye ridges of the buffalo trunkfish. Local pressure minima were present at the buffalo trunkfish eye ridge at the $1 \%$ transect at positive pitch angles (see port 7 highlighted in the blue box). At pitch angles $>-6^{\circ}$ and $\leqslant-24^{\circ}$, there was also evidence of local pressure minima at the eye ridge, but not at moderate $\left(-10\right.$ to $\left.-20^{\circ}\right)$ pitch angles. However, local pressure minima could well have been present at port 8 at these moderate negative pitch angles (port 8 was defective and thus was not included in the analysis).

At positive pitch angles, dorsal pressure was lowest at the eye ridge for all three boxfishes (see 1\% transects, Figs 5-7), which is where concentrated vorticity was observed. At negative pitch angles in regions where concentrated dorsal vorticity was detected, dorsal pressure was not always lowest at the eye ridge, however. For example, dorsal pressure was lowest at port 18 (maximum girth) for pitch angles of 0 to $-16^{\circ}$ and at port 44 (posterior region of carapace) for pitch angles of -22 to $-30^{\circ}$ in the spotted boxfish (Fig. 5). For the scrawled cowfish, dorsal pressure was lowest at port 21 (maximum girth) for pitch angles $\leqslant-10^{\circ}$ and for the buffalo trunkfish, dorsal pressure was lowest at port 17 (maximum girth) for pitch angles $\leqslant-12^{\circ}$ (Figs 6, 7).

Dorsal pressures at the posteriormost regions of the carapace 
A

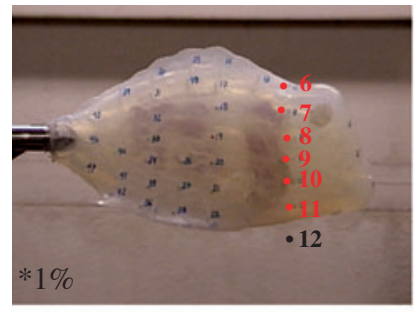

B

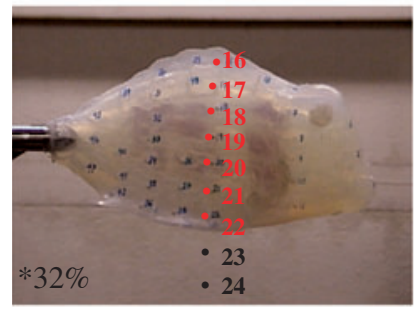

C

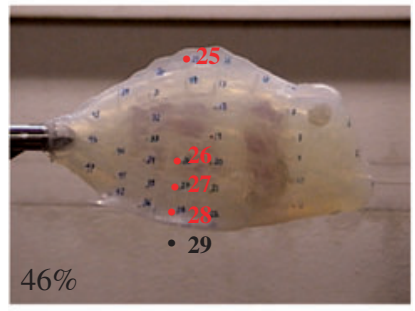

D

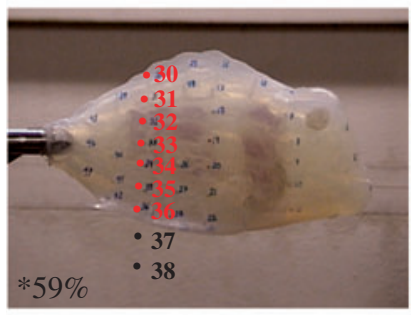

E

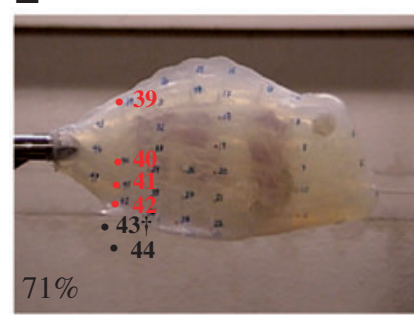

$\mathrm{F}$

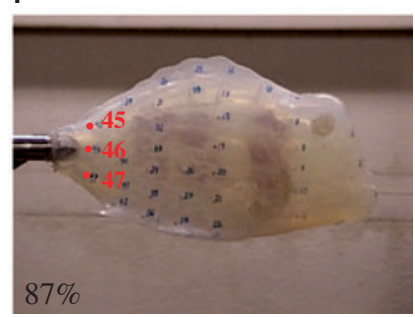

Positive pitch angles
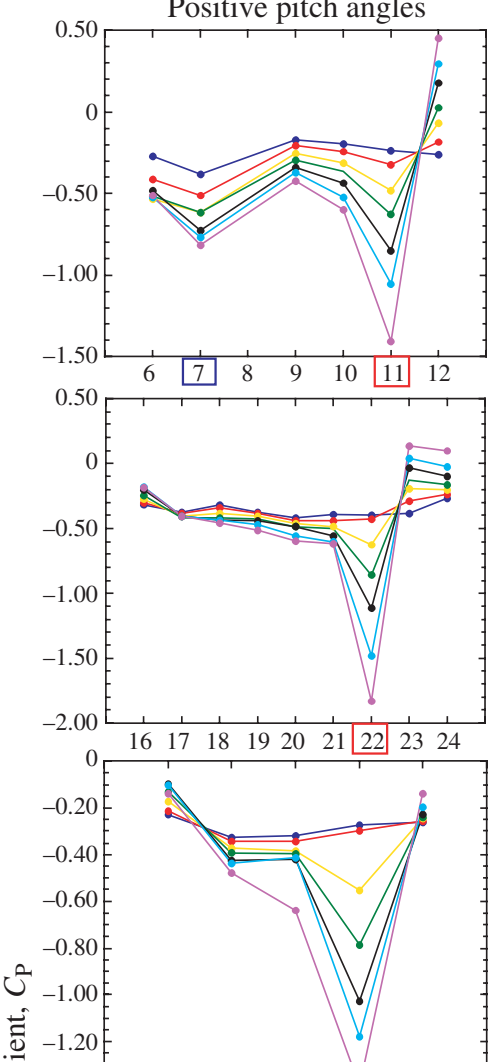

.

离
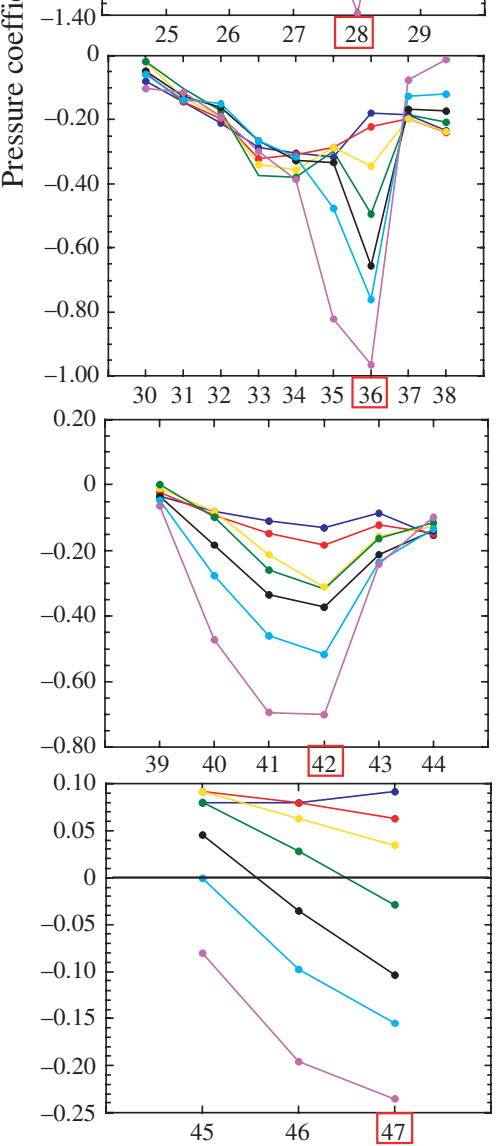

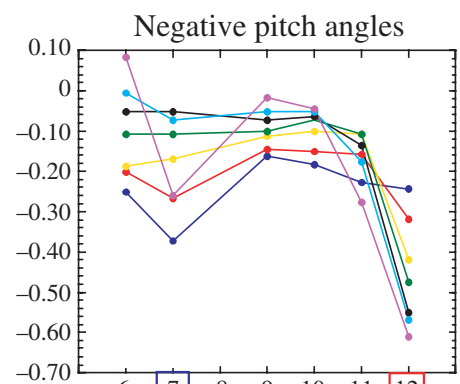

\begin{tabular}{cc|c|cccc|c|c|}
-0.70 & 1 & 6 & 7 & 8 & 9 & 10 & 11 & 12 \\
\hline
\end{tabular}

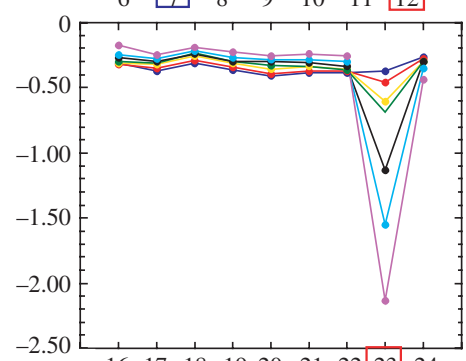

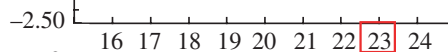

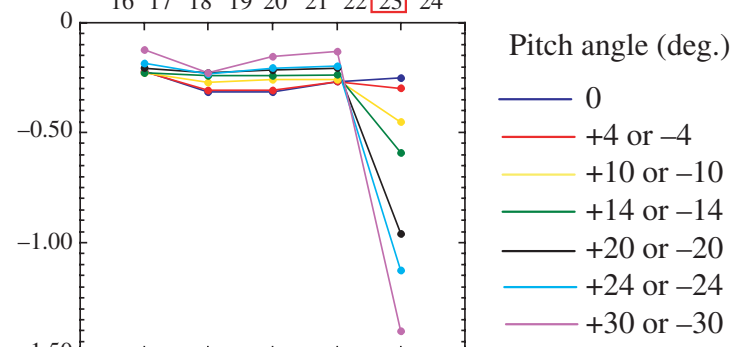

Pressure port 
were most consistently below ambient pressure in the spotted boxfish, especially at negative pitch angles (see port 44 for spotted boxfish and port 45 for scrawled cowfish and buffalo trunkfish). With few exceptions, ambient pressure $\left(C_{\mathrm{p}}=0\right)$ occurred between ports positioned immediately anterior and posterior to the eye at negative pitch angles. However, at positive pitch angles $>10^{\circ}$, ambient pressure did not occur consistently between ports positioned immediately anterior and posterior to the eye for the spotted boxfish and buffalo trunkfish. Only in the scrawled cowfish was ambient pressure consistently detected between the ports.

\section{Force balance measurements}

No obvious stall, i.e. the condition at which lift does not grow with increased pitch angle, occurred at any of the pitch angles examined for the three boxfishes (Fig. 8A). The absence of stall at pitch angles of $\pm 30^{\circ}$ is characteristic of delta wings, as is the general shape of the lift coefficient curves for all three boxfishes. Lift coefficients were closest to a value of 0 at $0^{\circ}$, where the coefficients were all positive. These positive coefficients are consistent with the observed downwash of flow detected at $0^{\circ}$ in DPIV experiments. At $0^{\circ}$, the spotted boxfish had a $C_{\mathrm{L}}=0.00908$, the scrawled cowfish had a $C_{\mathrm{L}}=0.00126$, and the buffalo trunkfish had a $C_{\mathrm{L}}=0.00620$. Drag coefficients were lowest at $-4^{\circ}$ for the spotted boxfish $\left(C_{\mathrm{D}}=0.272\right),-2^{\circ}$ for the scrawled cowfish $\left(C_{\mathrm{D}}=0.125\right)$ and $-2^{\circ}$ for the buffalo trunkfish $\left(C_{\mathrm{D}}=0.101\right)$ (Fig. $\left.8 \mathrm{~B}\right)$. Generally, nose-down and nose-up pitching moment coefficients $C_{\mathrm{M}}$ occurred at positive and negative pitch angles, respectively. The transition between nose-up and nose-down pitching moments occurred at approximately $+2^{\circ}$ for the spotted boxfish and between 0 and $-2^{\circ}$ for the scrawled cowfish and buffalo trunkfish (Fig. 8C). Furthermore, the absolute magnitude of $C_{\mathrm{M}}$ increased as pitch angles became more positive and more negative.

\section{Correlations of vortex strength, pressure and pitch moments}

Clear correlations among vortex circulation, pressure coefficients and pitching moment coefficients were detected at the posterior edge of the carapace (Table 1). As pitch angle deviated farther from $0^{\circ}$ and circulation of vortices at the posterior edge of carapace increased, pressure coefficients and

Fig. 7. Pressure coefficients $\left(C_{\mathrm{p}}\right)$ plotted as a function of location along various dorso-ventral transects $(\mathrm{A}-\mathrm{F})$ on the buffalo trunkfish model positioned at positive (left) and negative (right) pitch angles. The locations of the pressure ports included in each graph are highlighted in images to the left of the graphs. The location of each dorso-ventral transect is expressed as a percentage of ventral keel length (measured from the anterior leading edge) in the lower lefthand corner of each image. Ports in red and black are located on the sides and ventrum of the model, respectively. ${ }^{\dagger}$ Denotes ports that were slightly out of alignment with other ports along the dorso-ventral transect; * represent transects that were considered in DPIV studies. Blue and red rectangles highlight ports where local pressure minima were consistently detected at dorsal and ventral locations, respectively. An ambient pressure line $\left(C_{\mathrm{p}}=0\right)$ was included in the most posteriorly located transect $(\mathrm{F})$. pitching moment coefficients increased in absolute magnitude. Moreover, changes in pressure and pitching moment coefficients at various pitch angles were highly correlated. As pressure coefficients deviated farther from ambient conditions, pitching moment coefficients increased farther from zero.

\section{Discussion \\ General findings}

Although three very different carapace morphologies were examined, several important similarities in how the bodies direct flows emerged. The carapaces of all three boxfishes generated vortical, spiral flows, most consistently in locations adjacent to the ventral keels, but also to varying degrees in dorsal locations at various pitch angles. In general, ventral vortices were stronger in terms of circulation than those detected in dorsal locations and had larger effects on localized pressures along the carapace, as was evident by the consistent detection of pronounced local pressure minima in areas adjacent to the ventral keels. Leading edge vortices (LEVs) developed first at the anterior edges of the ventral keels and then grew in circulation as they traveled posteriorly along the body until the posterior edge of the carapace, where boundary layer vorticity fed into the LEV and where the LEV began to leave the body. Additional growth in circulation of the LEVs also was observed at the caudal peduncle, which probably occurred as even more of the body's boundary layer vorticity was shed into the LEVs. As pitch angles increased from $0^{\circ}$ in the positive direction, LEVs with stronger peak vorticity and circulation developed above ventral keels, often within concavities that served to hold vortices in place, and reached maximum strength (while attached) at postero-lateral regions of the carapace. As pitch angles increased from $0^{\circ}$ in the negative direction, LEVs with stronger peak vorticity and circulation developed below ventral keels often within adjacent concavities, reaching maximum strength (while attached) at postero-ventral regions of the carapace.

Low pressure correlated well with regions of concentrated vorticity. Local pressure minima were observed when vortex cores were close to the carapace surface, and pressure decreased as pitch angles deviated from $0^{\circ}$, either in the negative or positive direction. This was expected based on Bernoulli's principle, which states that higher local speeds result in lower static pressure. With this principle in mind, low static pressure should be detected along the carapace when the core of a vortex, a region in which flow speeds are higher than the surrounding fluid, is close to the surface. As the vortex intensifies in strength and local speeds increase, surface pressures should become increasingly more negative and conspicuous (McCormack and Crane, 1973; Tritton, 1998; Vogel, 2003).

One fairly consistent finding among the boxfishes was that surface pressures generally did not continue to decrease along antero-posterior transects where regions of concentrated vorticity were detected and were found to increase in circulation. Instead, lowest pressures along these transects 
often were detected in more anterior regions, such as at maximum girth. This is because the vortex cores began to move away from the body in posterior locations, thus having less effect on surface pressures. (Of course, it should be noted that the vortex cores are also larger in posterior regions and thus affect larger areas, as observed in the spotted boxfish and buffalo trunkfish.) Nonetheless, based on pressure coefficients, posterior vortices associated with the ventral keels appeared to
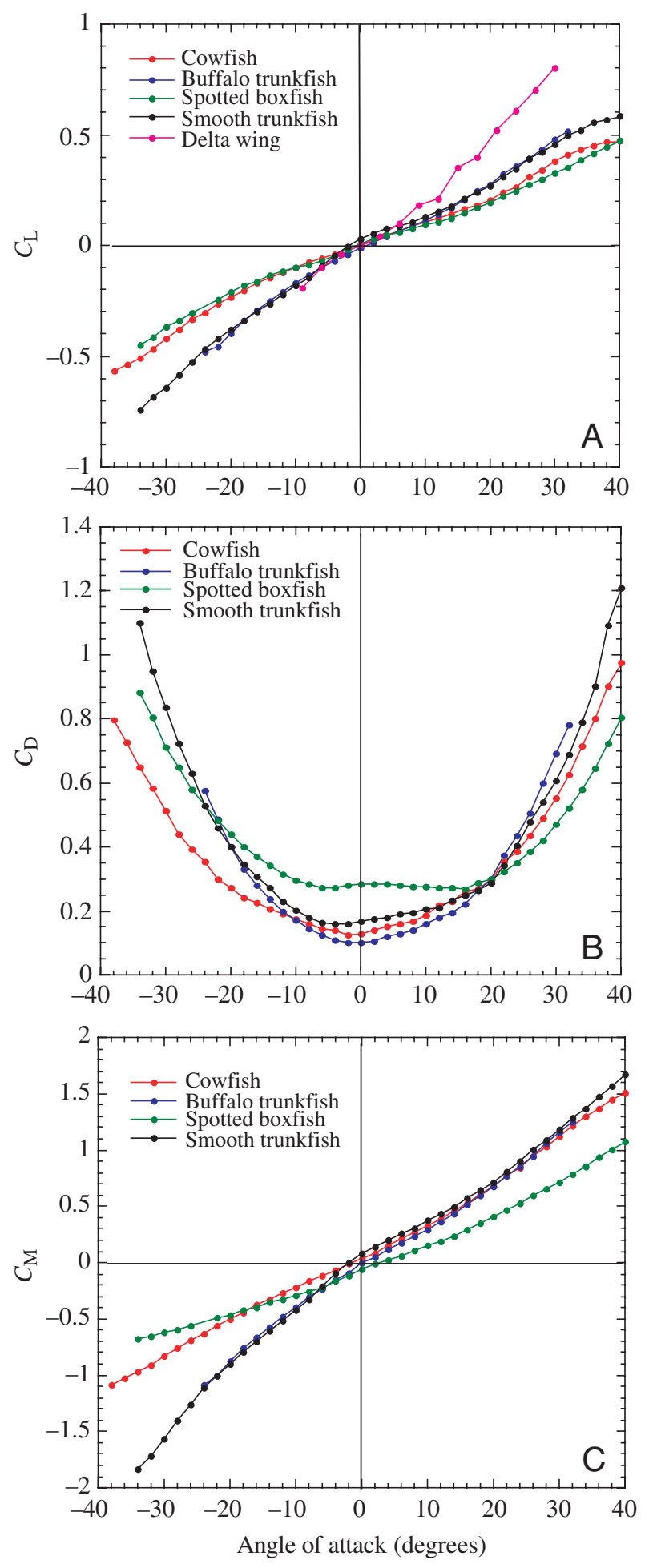

energize flow close to the carapace surface and presumably prevented flow separation in posterior regions of the carapace (pressure coefficients were $<0$ in postero-ventral regions). This finding is important because it suggests that fin motion is not necessary to keep flow attached to the body. Interestingly, all of the patterns described above also were detected in the smooth trunkfish Lactophrys triqueter, a boxfish with a broadly triangular cross-section, but with no significant ornamentation (Bartol et al., 2003).

\section{Species-specific differences}

Several noteworthy differences in flow patterns around the three boxfishes were observed. The most obvious difference was that dorsal vortices most consistently formed, grew posteriorly, and were of the highest magnitude in the spotted boxfish compared with the other boxfishes. This is not surprising given that the spotted boxfish has two widely separated dorsal keels that extend longitudinally from the eye ridges to the posterior edge of the carapace. These keels, which become more acute in posterior regions, provide structure for vorticity build-up and intensification, similar to that provided by the ventral keels (Bartol et al., 2002). In fact, vortices around the dorsal keels energized flow close to the carapace surface and prevented flow separation in postero-dorsal regions of the carapace, just as ventral vortices did. In the other boxfishes, the eye ridges do not extend posteriorly as sharp lateral keels. Instead the eye ridges terminate along the body, while the single dorsal keel forms between the ridges.

The effects of localized dorsal vortex formation in spotted boxfish were most pronounced at high positive and negative pitch angles, when strong attached vortices and low pressures were detected at the posteriormost regions of the carapace. At more anterior regions (e.g. $40 \%$ transect), DPIV and pressure results indicate that attached concentrated vorticity migrates above and below the dorsal keel at certain pitch angles. This may occur because the vortex shedding point on the eye ridge moves as the pitch angle changes, such that the vortex slides up and down. The convexity of the dorsum and/or rounded nature of the keel at maximum girth (Bartol et al., 2002) also may facilitate vortex migration. Interestingly, there was significantly less vortex migration at the posterior quarter of the carapace, where the dorsal keels sharpen, and there is a pronounced region of concavity above and below the keel (Bartol et al., 2002). These features facilitate vortex generation and retention during pitching, as evident by the consistent

Fig. 8. Lift coefficients $C_{\mathrm{L}}(\mathrm{A})$, drag coefficients $C_{\mathrm{D}}(\mathrm{B})$, and pitching moment coefficients about the center of mass $C_{\mathrm{M}}(\mathrm{C})$ for four boxfishes (smooth trunkfish, buffalo trunkfish, scrawled cowfish and spotted boxfish) positioned at various pitch angles. In $\mathrm{A}, C_{\mathrm{L}}$ values for a delta wing of similar aspect ratio $(0.83)$ to that of the boxfishes are also depicted. Delta wing data are from Schlichting and Truckenbrodt (1969), and smooth trunkfish data are from Bartol et al. (2003). Positive $C_{M}$ values indicate a nose-down pitching moment about the center of mass, whereas negative $C_{\mathrm{M}}$ values indicate a noseup pitching moment about the center of mass. 
Table 1. Correlations of vortex circulation, pressure coefficients and pitching moment coefficients for various boxfishes

\begin{tabular}{lccccccc}
\hline Boxfish & Comparison & Count & Z-value & $P$-value & $r$ & $95 \%$ lower C.I. & 95\% upper C.I. \\
\hline SB & C vs $P$ & 7 & 4.35 & $<0.0001$ & 0.974 & 0.832 & 0.996 \\
SB & C vs $M$ & 7 & 4.61 & $<0.0001$ & 0.980 & 0.868 & 0.997 \\
SB & $P$ vs $M$ & 7 & 3.84 & 0.0001 & 0.958 & 0.735 & 0.994 \\
SC & $C$ vs $P$ & 7 & 3.72 & 0.0002 & 0.953 & 0.706 & 0.993 \\
SC & $C$ vs $M$ & 7 & 4.84 & $<0.0001$ & 0.984 & 0.894 & 0.924 \\
SC & $P$ vs $M$ & 7 & 5.19 & $<0.0001$ & 0.989 & 0.796 & 0.998 \\
BT & $C$ vs $P$ & 7 & 4.13 & $<0.0001$ & 0.968 & 0.961 & 0.754 \\
BT & $C$ vs $M$ & 6 & 5.35 & $<0.0001$ & 0.996 & 0.997
\end{tabular}

SB, spotted boxfish; SC, scrawled cowfish; BT, buffalo trunkfish.

$C$, vortex circulation; $P$, pressure coefficient; $M$, pitching moment coefficient; $r$, correlation coefficient.

Circulation of vortices found above (positive pitch angles) and below (negative pitch angles) the ventral keels at the posterior edge of the carapace were used in the correlations. Pressure coefficients at ports just above (positive pitch angles) or just below (negative pitch angles) the ventral keels, at the posterior edge of the carapace in locations where vortex circulation was measured, were used in the correlations. Data at pitch angles of $30,20,10,0,-10,-20$ and $-30^{\circ}$ were considered in correlations for the spotted boxfish and scrawled cowfish. These angles also were considered in the buffalo trunkfish for correlations between vortex circulation and pressure coefficient, but $-30^{\circ}$ was omitted from correlations involving pitching moment coefficients because of a lack of data.

observation of strong vortices above and below the keels in the posteriormost carapace locations at positive and negative pitch angles, respectively. The effect of these vortices on the surface was apparent in pressure data for negative pitch angles (unfortunately, a lack of pressure ports above the dorsal keels in these posterior regions precluded an examination of surface pressures at positive pitch angles).

Dorsal vortices also formed and were retained around the carapace of the scrawled cowfish, albeit to a much lesser degree than those detected in the spotted boxfish. As indicated above, the scrawled cowfish does not have two dorsal keels that extend along the length of the carapace. However, it does have two prominent anterior horns, eye ridges, and strong lateral convexity that extends along the sides of the carapace, producing a somewhat pentagonal cross-section (Bartol et al., 2002). The horns and eye ridges generated vortices, and these vortices remained attached to the body either above or below regions of lateral convexity. The convex regions lack sharp edges, as in spotted boxfish, but provide perhaps enough geometry to retain and even facilitate minor vortex circulation growth as boundary layer vorticity from the body feeds into the dorsal LEV. The observance of low pressure in areas adjacent to regions of convexity is consistent with the DPIV findings. The absence of sharp keels and regions of dorsal concavity to corral vorticity led to high migratory movement of lateral vortices. Moreover, unlike the spotted boxfish, dorsal vortices in the scrawled cowfish do not appear to be strong enough to energize flow sufficiently to prevent flow detachment near the posterior edge of the carapace (pressure coefficients were often greater than 0 ).

The ventral keel extensions in the scrawled cowfish are important flow-directing features. These extensions, which are close to the body and parallel to the longitudinal body axis, stimulated vortex formation and kept vortices close to the body. Based on the results of this study, these keel extensions, together with a very pronounced concave ventrum, were especially effective at facilitating attached vortex growth and producing low pressures at the posteriormost sections of the carapace at negative pitch angles, especially those of -10 to $-14^{\circ}$.

The buffalo trunkfish generated well-developed ventral vortices but produced minimal dorsal vorticity posterior to the eye ridge. This is similar to results from a smooth trunkfish, which has a similar cross-section (Bartol et al., 2003). The buffalo trunkfish has ventral keel extensions like scrawled cowfish. However, the keel extensions are shaped differently in the two species. Rather than extending parallel to the longitudinal axis of the fish and being very close to the body, the keel extensions in the buffalo trunkfish extend more laterally and are farther away from the body. Consequently, the keel extensions in the buffalo trunkfish do not appear to keep vortices as close to the carapace as in the scrawled cowfish. Although not detected in this study, it should be noted that these more laterally extended keels may facilitate beneficial vortex production and produce low pressure along other regions of the carapace that were not sampled (e.g. at the keel extension itself or at regions more aligned with the tip of the extension).

The scrawled cowfish, buffalo trunkfish and smooth trunkfish all have more triangular cross-sections and sharper ventral keels than the trapezoidal spotted boxfish (Bartol et al., 2002). Consequently, the vortices that developed around the ventral keels of the cowfish and trunkfishes were more ordered and had more pronounced effects on surface pressures. This is clear when examining pressure distributions. In scrawled cowfish, buffalo trunkfish and smooth trunkfish, conspicuous pressure minima were observed adjacent to the ventral keels, whereas in the spotted boxfish, low-pressure zones were less apparent. 


\section{Lift coefficients and delta wings}

The vortical flow patterns observed around the ventral keels of the three boxfishes resemble flows around delta wings. Delta wings are symmetrical, triangular wings designed to fly at subsonic or supersonic speeds. Some examples of aircraft with delta wings are the Concorde SST, Lockhead CL-8233, North American XB-70 Valkyrie supersonic bomber, and even the space shuttle. In delta-winged aircraft, a coiled (spiral) vortex sheet with a core of high vorticity forms at the leading edge of the wing and grows posteriorly along the wing, inducing a field of low pressure above the wing that produces lift (Bertin and Smith, 1989). Since lift is created with vortices as opposed to bound circulation, as in conventional wings, stall, i.e. vortex bursting, does not occur until high pitch angles. The ventrum of boxfishes differs from delta wings in that its maximum width/span occurs close to the middle of the fish as opposed to the posterior end, as in delta wings. Nonetheless, vortex formation is similar. Based on DPIV results, spiral vortices like those observed in delta wings form along the ventral keels and grow in strength as they travel posteriorly and vorticity feeds into the vortices. We can be confident that spirality is present in boxfishes because (a) vortices become stronger moving downstream and (b) each planar slice sampled along the body reveals steady, time-independent vorticity. These conditions can only occur if added vorticity per unit time is swept downstream by the axial component of velocity (i.e. there is axial streamwise flow).

Given their differences in shape, it is not surprising that boxfishes and delta wings have different lift coefficient magnitudes. However, the shapes of the curves, whereby there is no evidence of stall at $\pm 30^{\circ}$, is further support that vortex generation is similar in boxfishes and delta wings. Interestingly, vortex flows above delta wings migrate vertically above the wing as they travel along the chord of the wing. Consequently pressures directly below the vortex cores increase posteriorly (Zohar and Er-El, 1988; Pashilkar, 2001). This was also observed along the bodies of boxfishes. Based on force measurements, lift coefficients were closest to 0 and slightly positive at $0^{\circ}$. This too is in agreement with DPIV data. DPIV results indicate that lowest vortex circulation occurs at pitch angles near $0^{\circ}$, and vortices are generated above ventrolateral keels at $0^{\circ}$, producing a downwash of flow in the wake. This downwash provides beneficial lift for counteracting negative buoyancy present in rigid-bodied ostraciids (Blake, 1977).

\section{Self-corrective trimming control}

The vortical flow patterns induced by the carapaces of the different boxfishes, which were similar around the ventral keels but variable in dorsal locations, all should produce trimming forces that self-correct for pitching, i.e. rotation in the vertical, head-up/down longitudinal plane. In ventral locations, attached vortices with the strongest peak vorticity and circulation developed posterior to the center of mass, which is located approximately at maximum girth (Fig. 1; Bartol et al., 2002). These vortices formed above (positive pitch angles) or below (negative pitch angles) ventral keels that extend laterally at an angle of $0-40^{\circ}$ relative to a horizontal axis when viewed in cross-section. Consequently, suction derived from the presence of a vortex above or below the ventral keels, which was evident as low pressure zones in pressure experiments, should act largely upward and posterior to the center of mass at positive pitch angles (which also occurs in delta wings) and downward and posterior to the center of mass at negative pitch angles.

In spotted boxfish, dorsal vortices, which have their greatest circulation and peak vorticity posterior to the center of mass, also should play an important role in self-correction. These vortices generally formed above the dorsal keels, which extend at an angle of $0-30^{\circ}$ relative to a horizontal axis when viewed in cross-section at positive pitch angles, and below them along the sides of the carapace at negative pitch angles. Low pressure derived from these vortices should produce forces that counteract pitching motions, like those in ventral regions. Vortices forming above and below convex regions in the scrawled cowfish and keel extensions in scrawled cowfish and buffalo trunkfish also contribute to self-righting forces that counteract pitching motions. However, these forces are substantially less than those associated with the main keels, because the vortices are of lower magnitude or act on a smaller surface area.

Pitching moments recorded in force balance experiments, which correlated statistically with both pressure and DPIV data, support the predictions above. Nose-down pitching moments occurred and became progressively stronger as pitch angles became more positive, and nose-up pitching moments occurred and became progressively stronger as pitch angles became more negative. At positive pitch angles, smooth trunkfish, buffalo trunkfish, and scrawled cowfish all produced similar self-correcting forces, with spotted boxfish producing self-correcting forces of lower magnitude. At negative pitch angles, smooth trunkfish and buffalo trunkfish produced strong self-correcting forces, while scrawled cowfish and spotted boxfish produced correcting forces of lower magnitude.

The trunkfishes are particularly effective at generating selfcorrecting forces because they have sharper ventral keels along most of their bodies than the other boxfishes and have broad lateral surface areas above and below the ventral keels onto which vortices may act (Bartol et al., 2002). The scrawled cowfish also has sharp ventral keels with a pronounced lateral lip in posterior regions, which certainly aids in generating selfcorrecting forces at positive pitch angles. However, at negative pitch angles, the narrow ventrum does not allow the two counter-rotating vortices forming below the ventral keels to develop in isolation as in the trunkfishes, which have broad bases. Instead ventral vortices interact with one another at negative pitch angles, and this may lower the magnitude of self-correcting forces. Lower magnitude dorsal vortices in posterior regions of the carapace at negative compared with positive pitch angles (see Fig. 4) also may contribute to the observed asymmetric pitching moment curve for scrawled cowfish. Based on the geometry of the spotted boxfish, which 
has four keels and broad regions both above the dorsal keels and below the ventral keels onto which vortices may act, we expected these fish to produce the strongest self-correcting forces. However, the less acute, often rounded, dorsal and ventral keels of the spotted boxfish simply did not produce vortices of the magnitude of those observed for the trunkfishes. Irrespective of the differences in the magnitude of the selfcorrecting forces and the distinctions in carapace morphologies among the boxfishes, the integrated effects of the flow patterns around the carapaces result in the production of self-correcting forces for pitching motions in all boxfishes. Moreover, the selfcorrecting couple is proportional to the angle to which the fish is perturbed from a horizontal swimming trajectory.

Although moments and pressure distributions at various yaw angles were not measured, DPIV results indicate that the carapaces of all three boxfishes also generate self-correcting forces for yawing, i.e. rotations in the left/right horizontal frontal plane. Stronger dorsal and ventral vortices clearly formed on the far-field as opposed to the near-field side of carapace, especially in areas adjacent to the dorsal and ventral keels, when the boxfishes were positioned at various yaw angles. These differences may be attributed in large part to changes in sweep angle. When the boxfish yaws, the near-field side effectively decreases in sweep angle, while the far-field side increases in sweep angle. In delta wings, the larger the sweep angle up to a reasonable maximum (e.g. $75^{\circ}$ ), the stronger the LEV (Bertin and Smith, 1989). Circulation of these far-field vortices increased posteriorly along the carapace, such that maximum vortex circulation occurred posterior to the center of mass. Vortex circulation and peak vorticity also increased as yaw angles increased. Suction derived from the presence of vortices at far-field locations of the carapace should act largely opposite the direction of the yaw and posterior to the center of mass, thus providing trimming forces that self-correct for yawing motions. As with pitching, the self-correcting couple is proportional to the angle to which the fish is perturbed.

\section{Role of the eye ridges}

In the three boxfishes examined in the present study and in the smooth trunkfish, regions of concentrated vorticity formed around the eye ridges, producing pressure distributions where ambient pressure occurs at the eye at low positive pitch angles and negative pitch angles. This may be beneficial for eye function because the eyeball is not deformed (i.e. lens of the eye is not pushed in or pulled out) as flow moves along the body, and thus the optical properties of the eye are not altered. Detection of ambient pressure around the eyes also has been reported in squid, bluefish and tuna (Aleyev, 1977; Dubois et al., 1974; Vogel, 1987). Interestingly, ambient pressure was most consistently produced around the eyes of the scrawled cowfish at positive and negative pitch angles, suggesting that the anterior horns may play an important role in controlling flows for eye function. This hypothesis, together with the investigation of flow/pressure field effects on the eyes of swimming fishes, merits further study. The eye ridges also generate some lift to counteract the nose-down pitching moment produced by the ventral keels at a $0^{\circ}$ pitch angle. This allows for more uniform lift production about the center of mass to counteract negative buoyancy while swimming in straight paths.

\section{Body-induced flows and their ecological implications}

The results of the present study indicate that the rigid keeled carapaces of boxfishes play an important role in the control of vorticity, producing flows and pressure distributions that comprise an efficient, self-correcting system for pitch and yaw. To date this self-correcting system has been documented in four morphologically distinct boxfishes: spotted boxfish, scrawled cowfish, buffalo trunkfish and smooth trunkfish (Bartol et al., 2003). This self-corrective trimming system is important for several reasons. First it damps large perturbations when swimming and when holding position while feeding on prey lodged in complex habitats. This damping is especially important in highly energetic turbulent waters where flows may frequently move boxfishes off their desired paths or positions. Second, it acts quickly and automatically and does not require neural processing, as in powered control systems (e.g. asymmetrical pectoral fin beats to correct a rolling moment). This is especially advantageous for unpredictable velocity fields, where accurate phasing of powered correction forces with perturbations is difficult and requires rapid neural processing. Without proper phasing, correction may even amplify disturbances through 'pilot induced error' (Webb, 1998, 2000, 2002). Third, maintenance of smooth swimming trajectories improves sensory acuity of both hostile (i.e. predators) and target objects because it reduces complexity of movement, a factor that enhances sensory perception in other animals (Land, 1999; Kramer and McLaughlin, 2001).

In addition to the advantages of the carapace and selfcorrecting system, there are some disadvantages. Although the keels presumably provide energy savings by providing counter-moments to pitching and yawing, it should also be noted that there are some energetic costs associated with the maintenance of keel tissue, although these costs are probably low relative to the energetic costs of swimming and metabolic costs of other physiological functions. The rigid, boxy carapace, although effective for producing self-correcting forces, also incurs high drag. The drag coefficients reported in this study are indeed high relative to other fishes (Blake, 1981, 1983). The most significant disadvantage of the self-correcting system, however, is that it increases costs for maneuverability. A self-correcting system by definition means that any change in course, whether voluntary or involuntary, is counteracted by the stabilizing mechanism. Consequently, it presumably requires more power and time to actuate turning maneuvers. This is consistent with Walker's finding (Walker, 2000) that one species of boxfish, Ostracion meleagris, has slower rates of turning than flexible-bodied fish.

Role of the fins

When considering stability control and maneuverability, it 
is important to consider flow interactions between the fins and body. Movements of the pectoral fins, which are located near the path of body-induced ventral vortex formation, will presumably affect body vortex magnitude and structure. For example, asymmetric pectoral fin movements could strengthen or weaken body-induced vortices on one side of the body relative to the other, producing favorable forces for lateral rotations. The dorsal fin also may intercept concentrated vorticity in postero-dorsal areas of the carapace, which was detected in all boxfishes, to improve its efficacy in producing stabilizing forces. The capture of vorticity by fins to improve thrust and swimming efficiency has been introduced in previous studies (Lighthill, 1970; Wu, 1971; Weihs, 1989) and has been modeled recently in tunas and danios (Zhu et al., 2002). The interactions of various fins with body-induced flows is the subject of ongoing studies in our laboratories.

\section{Concluding remarks}

The carapaces of boxfishes, which vary in cross-sectional shape, longitudinal features and ornamentation, play an important role in hydrodynamic stability. The ventral keels of boxfishes, which often have adjacent regions of concavity for holding vorticity in place, and the dorsal keels in the spotted boxfish, are vortex generators, producing flows similar to those around the leading edges of delta-winged aircraft. The resulting surface pressures produced by the flows contribute to integrated forces that self-correct for pitching and probably yawing movements, allowing these fishes to swim in remarkably straight paths. The evolution of such a sophisticated self-correcting system in this group of fishes is beneficial for the varied environmental conditions these animals face within their natural habitats. This system is useful for steady swimming in highly turbulent waters where countermoments for pitching and yawing are required, or perhaps for station-holding in highly variable flows during feeding on coral inhabitants. Increased understanding of this self-correcting system, especially in regards to how the fins interact with bodyinduced flows, promises to reveal novel mechanisms for stability control.

We thank R. M. Alexander and P. Krueger for valuable intellectual input, M. McNitt-Gray, J. Carnahan, B. Valiferdowsi, and P. Masson for aid during model construction, D. Lauritzen and S. Bartol for assistance during data collection, and D. Dabiri, L. Zuhal, and D. Jeon for technical assistance. We especially thank M. Grosenbaugh at Woods Hole Oceanographic Institution for providing office space during the writing phase of this paper and two anonymous reviewers for constructive comments. Buffalo trunkfish and scrawled cowfish were collected by W. Rosado, Department of Marine Science, University of Puerto Rico-Mayaquez. Financial support of the Office of Naval Research under grants N00014-96-1-0607 and N00014-02-1-0180 (to M.S.G. and M.G.) is gratefully acknowledged.

\section{References}

Aleyev, Y. G. (1977). Nekton. The Hague: Junk.

Bartol, I. K., Gharib, M., Weihs, D., Webb, P. W., Hove, J. R. and Gordon, M. S. (2003). Hydrodynamic stability of swimming in ostraciid fishes: role of the carapace in the smooth trunkfish Lactophrys triqueter (Teleostei: Ostraciidae). J. Exp. Biol. 206, 725-744.

Bartol, I. K., Gordon, M. S., Gharib, M., Hove, J. R., Webb, P. W. and Weihs, D. (2002). Flow patterns around the carapaces of rigid-bodied, multi-propulsor boxfishes (Teleostei: Ostraciidae). Integ. Comp. Biol. 42, 971-980.

Bertin, J. J. and Smith, M. L. (1989). Aerodynamics for Engineers, 2nd edn. Englewood Cliffs: Prentice Hall.

Blake, R. W. (1977). On ostraciiform locomotion. J. Mar. Biol. Assn UK 57, 1047-1055.

Blake, R. W. (1981). Mechanics of ostraciiform propulsion. Can J. Zool. 59, 1067-1071.

Blake, R. W. (1983). Fish Locomotion. Cambridge: Cambridge University Press.

Dubois, A. B., Cavagna, G. A. and Fox, R. S. (1974). Pressure distribution of the body surface of swimming fish. J. Exp. Biol. 60, 581-591.

Gordon, M. S., Hove, J. R., Webb, P. W. and Weihs, D. (2000). Boxfishes as unusually well-controlled autonomous underwater vehicles. Physiol. Biochem. Zool. 73, 663-671.

Hove, J. R., O'Bryan, L. M., Gordon, M. S., Webb, P. W. and Weihs, D. (2001). Boxfishes (Teleostei: Ostraciidae) as a model system for fishes swimming with many fins: kinematics. J. Exp. Biol. 204, 1459-1471.

Kramer, D. L. and McLaughlin, R. L. (2001). The behavioral ecology of intermittent locomotion. Amer. Zool. 41, 137-153.

Land, M. F. (1999). Motion and vision: why animals move their eyes. $J$. Comp. Physiol. A 185, 341-352.

Lighthill, M. J. (1970). Aquatic animal propulsion of high hydromechanical efficiency. J. Fluid Mech. 44, 265-301.

Lisoski, D. (1993). Nominally 2-dimensional flow about a normal flat plate. $\mathrm{PhD}$ dissertation, California Institute of Technology, USA.

McCormack, P. D. and Crane, L. (1973). Physical Fluid Dynamics. New York: Academic Press.

Pashilkar, A. A. (2001). Surface pressure model for simple delta wings at high pitch angles. Sadhana 26, 495-515.

Raffel, M., Willert, C. E. and Kompenhans, J. (1998). Particle Image Velocimetry: A Practical Guide. Berlin: Springer-Verlag.

Schlichting, H. and Truckenbrodt, E. (1969). Aerodynamik des Flugzeuges, Vol. 2, Ch. 7. Berlin: Springer-Verlag.

Tritton, D. J. (1998). Physical Fluid Dynamics, 2nd edn. Oxford: Clarendon Press.

Tyler, J. C. (1980). Osteology, phylogeny, and higher classification of the fishes of the order Plectognathi (Tetraodontiformes). NOAA Tech. Rep. NMFS Circular 434, 1-422.

Vogel, S. (1987). Flow-assisted mantle cavity refilling in jetting squid. Biol. Bull. 172, 61-68.

Vogel, S. (2003). Comparative Biomechanics: Life's Physical World. Princeton: Princeton University Press.

Walker, J. A. (2000). Does a rigid body limit maneuverability? J. Exp. Biol. 203, 3391-3396.

Webb, P. W. (1998). Entrainment by river chub Nocomis micropogon and smallmouth bass Micropterus dolomieu on cylinders. J. Exp. Biol. 201, 2403-2412.

Webb, P. W. (2000). Maneuverability versus stability? Do fish perform well in both? 1st Int. Symp. Aqua Bio-mech./Int. Semin. Aqua Bio-mech. 1, 2129.

Webb, P. W. (2002). Control of posture, depth, and swimming trajectories of fishes. Integ. Comp. Biol. 42, 94-101.

Weihs, D. (1989). Design features and mechanics of axial locomotion in fish. Amer. Zool. 29, 151-160.

Willert, C. E. and Gharib, M. (1991). Digital particle image velocimetry. Exp. Fluids 10, 181-193.

Wu, T. Y. (1971). Hydromechanics of swimming propulsion. Part 3. Swimming and optimum movements of slender fish with side fins. J. Fluid Mech. 46, 545-568.

Zhu, Q., Wolfgang, M. J., Yue, D. K. P. and Triantafyllou, M. S. (2002). Three-dimensional flow structures and vorticity control in fish-like swimming. J. Fluid Mech. 468, 1-28.

Zohar, Y. and Er-El, J. (1988). Influence of the aspect ratio on the aerodynamics of the delta wing at high angle of attack. J. Aircraft 25, 200205. 